\title{
American Options, Multi-armed Bandits, and Optimal Consumption Plans: A Unifying View
}

\author{
By Peter Bank and Hans Föllmer*
}

\begin{abstract}
In this survey, we show that various stochastic optimization problems arising in option theory, in dynamical allocation problems, and in the microeconomic theory of intertemporal consumption choice can all be reduced to the same problem of representing a given stochastic process in terms of running maxima of another process. We describe recent results of Bank and El Karoui (2002) on the general stochastic representation problem, derive results in closed form for Lévy processes and diffusions, present an algorithm for explicit computations, and discuss some applications.
\end{abstract}

AMS 2000 subject classification. 60G07, 60G40, 60H25, 91B16, $91 \mathrm{~B} 28$.

Key words and phrases. American options, Gittins index, multi-armed bandits, optimal consumption plans, optimal stopping, representation theorem, universal exercise signal.

* Humboldt University of Berlin. Support of Deutsche Forschungsgemeinschaft through SFB 373, "Quantification and Simulation of Economic Processes", and DFG-Research Center "Mathematics for Key Technologies" (FZT 86) is gratefully acknowledged. 


\section{Introduction}

At first sight, the optimization problems of exercising an American option, of allocating effort to several parallel projects, and of choosing an intertemporal consumption plan seem to be rather different in nature. It turns out, however, that they are all related to the same problem of representing a stochastic process in terms of running maxima of another process. This stochastic representation provides a new method for solving such problems, and it is also of intrinsic mathematical interest. In this survey, our purpose is to show how the representation problem appears in these different contexts, to explain and to illustrate its general solution, and to discuss some of its practical implications.

As a first case study, we consider the problem of choosing a consumption plan under a cost constraint which is specified in terms of a complete financial market model. Clearly, the solution depends on the agent's preferences on the space of consumption plans, described as optional random measures on the positive time axis. In the standard formulation of the corresponding optimization problem, one restricts attention to absolutely continuous measures admitting a rate of consumption, and the utility functional is a time-additive aggregate of utilities applied to consumption rates. However, as explained in Hindy, Huang, and Kreps (1992), such time-additive utility functionals have serious conceptual deficiencies, both from an economic and from a mathematical point of view. As an alternative, Hindy, Huang, and Kreps (1992) propose a different class of utility functionals where utilities at different times depend on an index of satisfaction based on past consumption. The corresponding singular control problem raises new mathematical issues. Under Markovian assumptions, the problem can be analyzed using the Hamilton-Jacobi-Bellman approach; see Hindy and Huang (1993) and Benth, Karlsen, and Reikvam (2001). In a general semimartingale setting, Bank and Riedel (2001) develop a different approach. They reduce the optimization problem to the problem of representing a given process $X$ in terms of running suprema of another process $\xi$ :

$$
X_{t}=\mathbb{E}\left[\int_{(t,+\infty]} f\left(s, \sup _{v \in[t, s)} \xi_{v}\right) \mu(d s) \mid \mathscr{F}_{t}\right] \quad(t \in[0,+\infty))
$$

In the context of intertemporal consumption choice, the process $X$ is specified in terms of the price deflator; the function $f$ and the measure $\mu$ reflect the structure of the agent's preferences. The process $\xi$ determines a minimal level of satisfaction, and the

optimal consumption plan consists in consuming just enough to ensure that the induced index of satisfaction stays above this minimal level. In Bank and Riedel (2001), the 
representation problem is solved explicitly under the assumption that randomness is modelled by a Lévy process.

In its general form, the stochastic representation problem (1) has a rich mathematical structure. It raises new questions even in the deterministic case, where it leads to a timeinhomogeneous notion of convex envelope as explained in Bank and El Karoui (2002). In discrete time, existence and uniqueness of a solution easily follow by backwards induction. The stochastic representation problem in continuous time is more subtle. In a discussion of the first author with Nicole El Karoui at an Oberwolfach meeting, it became clear that it is closely related to the theory of Gittins indices in continuous time as developed by El Karoui and Karatzas (1994).

Gittins indices occur in the theory of multi-armed bandits. In such dynamic allocation problems, there is a number of parallel projects, and each project generates a specific stochastic reward proportional to the effort spent on it. The aim is to allocate the available effort to the given projects so as to maximize the overall expected reward. The crucial idea of Gittins (1979) consists in reducing this multi-dimensional optimization problem to a family of simpler benchmark problems. These problems yield a performance measure, now called the Gittins index, separately for each project, and an optimal allocation rule consists in allocating effort to those projects whose current Gittins index is maximal. Gittins (1979) and Whittle (1980) consider a discrete-time Markovian setting, Karatzas (1984) and Mandelbaum (1987) extend the analysis to diffusion models. El Karoui and Karatzas (1994) develop a general martingale approach in continuous time. One of their results shows that Gittins indices can be viewed as solutions to a representation problem of the form (1). This connection turned out to be the key to the solution of the general representation problem in Bank and El Karoui (2002). This representation result can be used as an alternative way to define Gittins indices, and it offers new methods for their computation.

As another case study, we consider American options. Recall that the holder of such an option has the right to exercise the option at any time up to a given deadline. Thus, the usual approach to option pricing and to the construction of replicating strategies has to be combined with an optimal stopping problem: Find a stopping time which maximizes the expected payoff. From the point of view of the buyer, the expectation is taken with respect to a given probabilistic model for the price fluctuation of the underlying. From the point of view of the seller and in the case of a complete financial market model, it involves the unique equivalent martingale measure. In both versions, the standard approach consists in identifying the optimal stopping times in terms of the Snell envelope of the given payoff process; see, e.g., Karatzas (1988). Following 
Bank (2003b), we are going to show that, alternatively, optimal stopping times can be obtained from a representation of the form (1) via a level crossing principle: A stopping time is optimal iff the solution $\xi$ to the representation problem passes a certain threshold. As an application in Finance, we construct a universal exercise signal for American put options which yields optimal stopping rules simultaneously for all possible strikes. This part of the paper is inspired by a result in El Karoui and Karatzas (1995), as explained in Section 1.1.3.

The reduction of different stochastic optimization problems to the stochastic representation problem (1) is discussed in Section 1. The general solution is explained in Section 2, following Bank and El Karoui (2002). In Section 3 we derive explicit solutions to the representation problem in homogeneous situations where randomness is generated by a Lévy process or by a one-dimensional diffusion. As a consequence, we obtain explicit solutions to the different optimization problems discussed before. For instance, this yields an alternative proof of a result by Mordecki (2002), Asmussen, Avram, and Pistorius (2002) and Boyarchenko and Levendorskiŭ (2002) on optimal stopping rules for perpetual American puts in a Lévy model.

Closed-form solutions to stochastic optimization problems are typically available only under strong homogeneity assumptions. In practice, however, inhomogeneities are hard to avoid, as illustrated by an American put with finite deadline. In such cases, closed-form solutions cannot be expected. Instead, one has to take a more computa-

tional approach. In Section 4, we present an algorithm developed in Bank (2003a) which explicitly solves the discrete-time version of the general representation problem (1). In the context of American options, for instance, this algorithm can be used to compute the universal exercise signal as illustrated in Figure 1.

Acknowledgement. We are obliged to Nicole El Karoui for introducing the first author to her joint results with Ioannis Karatzas on Gittins indices in continuous time; this provided the key to the general solution in Bank and El Karoui (2002) of the representation result discussed in this survey. We would also like to thank Christian Foltin for helping with the $\mathrm{C}++$ implementation of the algorithm presented in Section 4 .

\section{Contents}

1 Reducing optimization problems to a representation problem 4

1.1 American options . . . . . . . . . . . . . . . . 5

1.1 .1 Optimal stopping via Snell envelopes . . . . . . . . . . . . . 5 
American Options, Multi-armed Bandits, and Optimal Consumption Plans

$1.1 .2 \quad$ A level crossing principle for optimal stopping . . . . . . . . . . . 7

1.1 .3 Universal exercise signals for American options. . . . . . . . . . . 14

1.2 Optimal consumption plans . . . . . . . . . . . . . . . . . . 20

1.3 Multi-armed bandits and Gittins indices . . . . . . . . . . . . . . . . . . 25

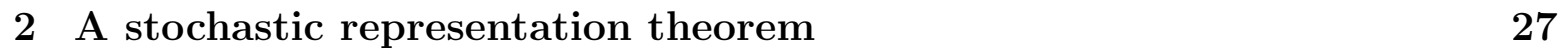

2.1 The result and its application . . . . . . . . . . . . . . . . . . 27

2.2 Proof of existence and uniqueness . . . . . . . . . . . . . . . . 31

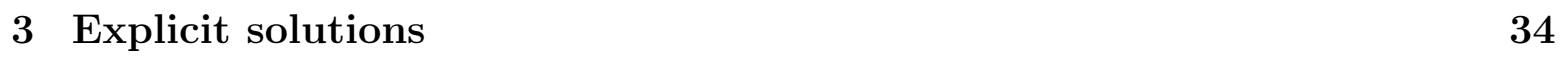

3.1 Lévy models . . . . . . . . . . . . . . . . . . . . . . . . . . . . . . . 34

3.1 .1 The perpetual American put . . . . . . . . . . . . . . . 35

3.1 .2 Optimal consumption . . . . . . . . . . . . . . . . . 36

3.2 Diffusion models . . . . . . . . . . . . . . . . . . . . . . . . 37

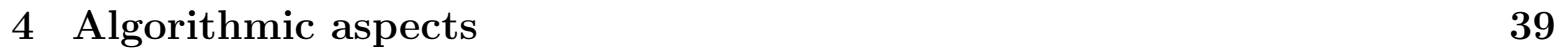

Notation. Throughout this paper we fix a probability space $(\Omega, \mathscr{F}, \mathbb{P})$ and a filtration $\left(\mathscr{F}_{t}\right)_{t \in[0,+\infty]}$ satisfying the usual conditions. By $\mathscr{T}$ we shall denote the set of all stopping times $T \geq 0$. Moreover, for a (possibly random) set $A \subset[0,+\infty], \mathscr{T}(A)$ will denote the class of all stopping times $T \in \mathscr{T}$ taking values in $A$ almost surely. For instance, given a stopping time $S$, we shall make frequent use of $\mathscr{T}((S,+\infty])$ in order to denote the set of all stopping times $T \in \mathscr{T}$ such that $T(\omega) \in(S(\omega),+\infty]$ for almost every $\omega$. For a given process $X=\left(X_{t}\right)$ we use the convention $X_{+\infty}=0$ unless stated otherwise.

\section{Reducing optimization problems to a representa- tion problem}

In this section we consider a variety of optimization problems in continuous time including optimal stopping problems arising in Mathematical Finance, a singular control problem from the microeconomic theory of intertemporal consumption choice, and the multi-armed bandit problem in Operations Research. We shall show how each of these different problems can be reduced to the same problem of representing a given stochastic process in terms of running suprema of another process. 


\subsection{American options}

An American option is a contingent claim which can be exercised by its holder at any time up to a given terminal time $\hat{T} \in(0,+\infty]$. It is described by a nonnegative, optional process $X=\left(X_{t}\right)_{t \in[0, \hat{T}]}$ which specifies the contingent payoff $X_{t}$ if the option is exercised at time $t \in[0, \hat{T}]$.

A key example is the American put option on a stock which gives its holder the right to sell the stock at a price $k \geq 0$, the so-called strike price, which is specified in advance. The underlying financial market model is defined by a stock price process $P=\left(P_{t}\right)_{t \in[0, \hat{T}]}$ and an interest rate process $\left(r_{t}\right)_{t \in[0, \hat{T}]}$. For notational simplicity, we shall assume that interest rates are constant: $r_{t} \equiv r>0$. The discounted payoff of the put option is then given by the process

$$
X_{t}^{k}=e^{-r t}\left(k-P_{t}\right)^{+} \quad(t \in[0, \hat{T}]) .
$$

\subsubsection{Optimal stopping via Snell envelopes}

The holder of an American put-option will try to maximize the expected proceeds by choosing a suitable exercise time. For a general optional process $X$, this amounts to the following optimal stopping problem:

Maximize $\mathbb{E} X_{T}$ over all stopping times $T \in \mathscr{T}([0, \hat{T}])$.

There is a huge literature on such optimal stopping problems, starting with Snell (1952); see El Karoui (1981) for a thorough analysis in a general setting. The standard approach uses the theory of the Snell envelope defined as the unique supermartingale $U$ such that

$$
U_{S}=\underset{T \in \mathscr{T}([S, \hat{T}])}{\operatorname{ess} \sup _{T}} \mathbb{E}\left[X_{T} \mid \mathscr{F}_{S}\right]
$$

for all stopping times $S \in \mathscr{T}([0, \hat{T}])$. Alternatively, the Snell envelope $U$ can be characterized as the smallest supermartingale which dominates the payoff process $X$. With this concept at hand, the solution of the optimal stopping problem can be summarized as follows; see Théorème 2.43 in El Karoui (1981):

Theorem 1.1 Let $X$ be a nonnegative optional process of class (D) which is uppersemicontinuous in expectation. Let $U$ denote its Snell envelope and consider its DoobMeyer decomposition $U=M-A$ into a uniformly integrable martingale $M$ and a predictable increasing process $A$ starting in $A_{0}=0$. Then

$$
\underline{T} \triangleq \inf \left\{t \geq 0 \mid X_{t}=U_{t}\right\} \quad \text { and } \quad \bar{T} \triangleq \inf \left\{t \geq 0 \mid A_{t}>0\right\}
$$


American Options, Multi-armed Bandits, and Optimal Consumption Plans

are the smallest and the largest stopping times, respectively, which attain

$$
\sup _{T \in \mathscr{T}([0, \hat{T}])} \mathbb{E} X_{T} .
$$

In fact, a stopping time $T^{*} \in \mathscr{T}([0, \hat{T}])$ is optimal in this sense iff

$$
\underline{T} \leq T^{*} \leq \bar{T} \quad \text { and } \quad X_{T^{*}}=U_{T^{*}} \quad \mathbb{P}-\text { a.s. }
$$

Remark 1.2 (i) Recall that an optional process $X$ is said to be of class (D) if $\left(X_{T}, T \in \mathscr{T}\right)$ defines a uniformly integrable family of random variables on $(\Omega, \mathscr{F}, \mathbb{P}) ;$ see, e.g., Dellacherie and Meyer (1980). Since we use the convention $X_{+\infty} \equiv 0$, an optional process $X$ will be of class (D) iff

$$
\sup _{T \in \mathscr{T}} \mathbb{E}\left|X_{T}\right|<+\infty
$$

and in this case the optimal stopping problem has a finite value.

(ii) As in El Karoui (1981), we call an optional process $X$ of class (D) uppersemicontinuous in expectation if for any monotone sequence of stopping times $T^{n}(n=1,2, \ldots)$ converging to some $T \in \mathscr{T}$ almost surely, we have

$$
\lim \sup \mathbb{E} X_{T^{n}} \leq \mathbb{E} X_{T} .
$$

$n$

In the context of optimal stopping problems, upper-semicontinuity in expectation is a very natural assumption.

Applied to the American put option on $P$ with strike $k>0$, the theorem suggests that one should first compute the Snell envelope

$$
U_{S}^{k}=\operatorname{esssup}_{T \in \mathscr{T}([S, \hat{T}])} \mathbb{E}\left[e^{-r T}\left(k-P_{T}\right)^{+} \mid \mathscr{F}_{S}\right] \quad(S \in \mathscr{T}([0, \hat{T}])) .
$$

and then exercise the option, e.g., at time

$$
\underline{T}^{k}=\inf \left\{t \geq 0 \mid U_{t}^{k}=e^{-r t}\left(k-P_{t}\right)^{+}\right\} .
$$

For a fixed strike $k$, this settles the problem from the point of view of the option holder. From the point of view of the option seller, Karatzas (1988) shows that the problem of pricing and hedging an American option in a complete financial market model amounts to the same optimal stopping problem, but in terms of the unique equivalent martingale measure $\mathbb{P}^{*}$ rather than the original measure $\mathbb{P}$. For a discussion of the incomplete case, see, e.g., Föllmer and Schied (2002). 


\subsubsection{A level crossing principle for optimal stopping}

In this section, we shall present an alternative approach to optimal stopping problems which is developed in Bank (2003b), inspired by the discussion of American options in El Karoui and Karatzas (1995). This approach is based on a representation of the underlying optional process $X$ in terms of running suprema of another process $\xi$. The process $\xi$ will take over the role of the Snell envelope, and it will allow us to characterize optimal stopping times by a level crossing principle.

Theorem 1.3 Suppose that the optional process $X$ admits a representation of the form

$$
X_{T}=\mathbb{E}\left[\int_{(T,+\infty]} \sup _{v \in[T, t)} \xi_{v} \mu(d t) \mid \mathscr{F}_{T}\right] \quad(T \in \mathscr{T})
$$

for some nonnegative, optional random measure $\mu$ on $([0,+\infty], \mathscr{B}([0,+\infty]))$ and some progressively measurable process $\xi$ with upper-right continuous paths such that

$$
\sup _{v \in[T(\omega), t)} \xi_{v}(\omega) 1_{(T(\omega),+\infty]}(t) \in L^{1}(\mathbb{P}(d \omega) \otimes \mu(\omega, d t))
$$

for all $T \in \mathscr{T}$.

Then the level passage times

$$
\underline{T} \triangleq \inf \left\{t \geq 0 \mid \xi_{t} \geq 0\right\} \quad \text { and } \quad \bar{T} \triangleq \inf \left\{t \geq 0 \mid \xi_{t}>0\right\}
$$

maximize the expected reward $\mathbb{E} X_{T}$ over all stopping times $T \in \mathscr{T}$.

If , in addition, $\mu$ has full support $\operatorname{supp} \mu=[0,+\infty]$ almost surely, then $T^{*} \in \mathscr{T}$ maximizes $\mathbb{E} X_{T}$ over $T \in \mathscr{T}$ iff

$$
\underline{T} \leq T^{*} \leq \bar{T} \mathbb{P}-\text { a.s. } \quad \text { and } \sup _{v \in\left[0, T^{*}\right]} \xi_{v}=\xi_{T^{*}} \mathbb{P}-\text { a.s. on }\left\{T^{*}<+\infty\right\}
$$

In particular, $\underline{T}$ is the minimal and $\bar{T}$ is the maximal stopping time yielding an optimal expected reward.

Proof : Use (4) and the definition of $\bar{T}$ to obtain for any $T \in \mathscr{T}$ the estimates

$$
\mathbb{E} X_{T} \leq \mathbb{E} \int_{(T,+\infty]} \sup _{v \in[0, t)} \xi_{v} \vee 0 \mu(d t) \leq \mathbb{E} \int_{(\bar{T},+\infty]} \sup _{v \in[0, t)} \xi_{v} \mu(d t)
$$

Choosing $T=\underline{T}$ or $T=\bar{T}$, we obtain equality in the first estimate since, for either choice, $T$ is a level passage time for $\xi$ so that

$$
\sup _{v \in[0, t)} \xi_{v}=\sup _{v \in[T, t)} \xi_{v} \geq 0 \quad \text { for all } \quad t \in(T,+\infty] .
$$


Since $T \leq \bar{T}$ in either case, we also have equality in the second estimate. Hence, both $T=\underline{T}$ and $T=\bar{T}$ attain the upper bound on $\mathbb{E} X_{T}(T \in \mathscr{T})$ provided by these estimates and are therefore optimal.

It follows that a stopping time $T^{*}$ is optimal iff equality holds true in both estimates occurring in (7). If $\mu$ has full support almost surely, it is easy to see that equality holds true in the second estimate iff $T^{*} \leq \bar{T}$ almost surely. Moreover, equality in the first estimate means exactly that (8) holds true almost surely. This condition, however, is equivalent to

$$
\lim _{t \downarrow T^{*}} \sup _{v \in[0, t)} \xi_{v}=\limsup _{t \searrow T^{*}} \xi_{t} \geq 0 \mathbb{P} \text {-a.s. on }\left\{T^{*}<+\infty\right\}
$$

which, by upper-right continuity of $\xi$, amounts to

$$
\sup _{v \in\left[0, T^{*}\right]} \xi_{v}=\xi_{T^{*}} \geq 0 \mathbb{P} \text {-a.s. on }\left\{T^{*}<+\infty\right\} \text {. }
$$

Equivalently:

$$
T^{*} \geq \underline{T} \mathbb{P} \text {-a.s. } \quad \text { and } \sup _{v \in\left[0, T^{*}\right]} \xi_{v}=\xi_{T^{*}} \geq 0 \mathbb{P} \text {-a.s. on }\left\{T^{*}<+\infty\right\} .
$$

Thus, optimality of $T^{*}$ is in fact equivalent to (6) if $\mu$ has full support almost surely.

Remark 1.4 (i) In Section 2, Theorem 2.2, we shall prove that an optional process $X=\left(X_{t}\right)_{t \in[0,+\infty]}$ of class (D) admits a representation of the form (4) if it is uppersemicontinuous in expectation. Moreover, Theorem 2.2 shows that we are free to choose an arbitrary measure $\mu$ from the class of all atomless, optional random measures on $[0,+\infty]$ with full support and finite expected total mass $\mathbb{E} \mu([0,+\infty])<$ $+\infty$. This observation will be useful in our discussion of American options in the next section.

(ii) The assumption that $\xi$ is upper-right continuous, i.e., that

$$
\xi_{t}=\limsup _{s \backslash t} \xi_{s}=\lim _{s \downarrow t} \sup _{v \in[t, s)} \xi_{v} \quad \text { for all } \quad t \in[0,+\infty) \quad \mathbb{P}-\text { a.s. },
$$

can be made without loss of generality. Indeed, since a real function $\xi$ and its

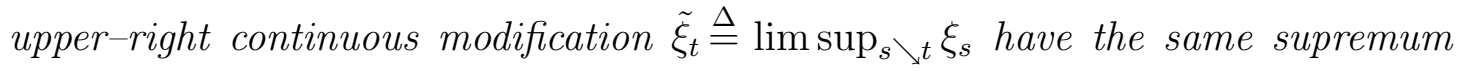
over sets of the form $[T, t)$, representation (4) is invariant under an upper-right continuous modification of the process $\xi$. The resulting process $\tilde{\xi}$ is again a progressively measurable process; see, e.g., from Théorème IV.90 of Dellacherie and Meyer (1975). 
American Options, Multi-armed Bandits, and Optimal Consumption Plans

(iii) The level crossing principle established in Theorem 1.3 also holds if we start at a fixed stopping time $S \in \mathscr{T}:$ A stopping time $T_{S}^{*} \in \mathscr{T}([S,+\infty])$ attains

$$
\operatorname{ess~sup}_{T \in \mathscr{T}([S,+\infty])} \mathbb{E}\left[X_{T} \mid \mathscr{F}_{S}\right]
$$

iff

$$
\underline{T}_{S} \leq T_{S}^{*} \leq \bar{T}_{S} \mathbb{P}-\text { a.s. and } \sup _{v \in\left[S, T_{S}^{*}\right]} \xi_{v}=\xi_{T_{S}^{*}} \text { on }\left\{T_{S}^{*}<+\infty\right\} \mathbb{P}-\text { a.s. },
$$

where $\underline{T}_{S}$ and $\bar{T}_{S}$ denote the level passage times

$$
\underline{T}_{S} \triangleq \inf \left\{t \geq S \mid \xi_{t} \geq 0\right\} \quad \text { and } \quad \bar{T}_{S} \triangleq \inf \left\{t \geq S \mid \xi_{t}>0\right\} .
$$

This follows as in the proof of Theorem 1.3, using conditional expectations instead of ordinary ones.

The preceding theorem reduces the optimal stopping problem to a representation problem of the form (4) for optional processes. In order to see the relation to the Snell envelope $U$ of $X$, consider the right continuous supermartingale $V$ given by

$$
V_{t} \triangleq \mathbb{E}\left[\int_{(t, \hat{T}]} \zeta_{s} \mu(d s) \mid \mathscr{F}_{t}\right]=\mathbb{E}\left[\int_{(0, \hat{T}]} \zeta_{s} \mu(d s) \mid \mathscr{F}_{t}\right]-\int_{(0, t]} \zeta_{s} \mu(d s)
$$

where

$$
\zeta_{s} \triangleq \sup _{v \in[0, s)} \xi_{v} \vee 0 \quad(s \in[0, \hat{T}])
$$

Since $V \geq X$, the supermartingale $V$ dominates the Snell envelope $U$ of $X$. On the other hand,

$$
V_{t}=\mathbb{E}\left[\int_{(\bar{T}, \hat{T}]} \zeta_{s} \mu(d s) \mid \mathscr{F}_{t}\right]=\mathbb{E}\left[X_{\bar{T}} \mid \mathscr{F}_{t}\right] \leq U_{t} \quad \text { on } \quad\{\bar{T} \geq t\},
$$

and so $V$ coincides with $U$ up to time $\bar{T}$. Is is easy to check that the stopping times $\underline{T}$ and $\bar{T}$ appearing in (2) and (5) are actually the same and that for any stopping $T^{*}$ with $\underline{T} \leq T^{*} \leq \bar{T}$ a.s., the condition $U_{T^{*}}=X_{T^{*}}$ in $(3)$ is equivalent to the condition $\sup _{v \in\left[0, T^{*}\right]} \xi_{v}=\xi_{T^{*}}$ in $(6)$.

A representation of the form (4) can also be used to construct an alternative kind of envelope $Y$ for the process $X$, as described in the following corollary. Part (iii) shows that $Y$ can replace the Snell envelope of Theorem 1.1 as a reference process for characterizing optimal stopping times. Parts (i) and (ii) are taken from Bank and El Karoui (2002). The process $Y$ can also be viewed as a solution to a variant of Skorohod's obstacle problem; see Remark 1.7 . 
Corollary 1.5 Let $\mu$ be a nonnegative optional random measure on $[0,+\infty]$ with full support $\operatorname{supp} \mu=[0,+\infty]$ almost surely and consider an optional process $X$ of class (D) with $X_{+\infty}=0 \mathbb{P}-$ a.s.

(i) There exists at most one optional process $Y$ of the form

$$
Y_{T}=\mathbb{E}\left[\int_{(T,+\infty]} \eta_{t} \mu(d t) \mid \mathscr{F}_{T}\right] \quad(T \in \mathscr{T})
$$

for some adapted, left continuous, nondecreasing process $\eta \in L^{1}(\mathbb{P} \otimes \mu)$ such that $Y$ dominates $X$, i.e.,

$$
Y_{T} \geq X_{T} \mathbb{P}-\text { a.s. for any } T \in \mathscr{T} \text {, }
$$

and such that $Y_{T}=X_{T} \mathbb{P}-$ a.s. for any point of increase $T$ of $\eta$.

(ii) If $X$ admits a representation of the form (4), then such a process $Y$ does in fact exist, and the associated increasing process $\eta$ is uniquely determined up to $\mathbb{P}_{-}$ indistinguishability on $(0,+\infty]$ via

$$
\eta_{t}=\sup _{v \in[0, t)} \xi_{v} \quad(t \in(0,+\infty])
$$

where $\xi$ is the progressively measurable process occurring in (4).

(iii) A stopping time $T^{*} \in \mathscr{T}$ maximizes $\mathbb{E} X_{T}$ over all $T \in \mathscr{T}$ iff

$$
\underline{T} \leq T^{*} \leq \bar{T} \quad \text { and } \quad Y_{T^{*}}=X_{T^{*}} \quad \mathbb{P}-\text { a.s. }
$$

where $\underline{T}$ and $\bar{T}$ are the level passage times

$$
\underline{T} \triangleq \inf \left\{t \in(0,+\infty] \mid \eta_{t} \geq 0\right\} \quad \text { and } \quad \bar{T} \triangleq \inf \left\{t \in(0,+\infty] \mid \eta_{t}>0\right\} .
$$

Remark 1.6 A stopping time $T \in \mathscr{T}$ is called a point of increase for a left-continuous increasing process $\eta$ if, $\mathbb{P}-$ a.s. on $\{0<T<+\infty\}, \eta_{T}<\eta_{t}$ for any $t \in(T,+\infty]$.

\section{ProOF :}

(i) In order to prove uniqueness, assume $\zeta \in L^{1}(\mathbb{P} \otimes \mu)$ is another adapted, left continuous and non-decreasing process such that the corresponding optional process

$$
Z_{T}=\mathbb{E}\left[\int_{(T,+\infty]} \zeta_{t} \mu(d t) \mid \mathscr{F}_{T}\right] \quad(T \in \mathscr{T})
$$


dominates $X$ and such that $Z_{T}=X_{T}$ for any time of increase $T \in \mathscr{T}$ for $\zeta$. For $\varepsilon>0$, consider the stopping times

$$
S^{\varepsilon} \triangleq \inf \left\{t \geq 0 \mid \eta_{t}>\zeta_{t}+\varepsilon\right\}
$$

and

$$
T^{\varepsilon} \triangleq \inf \left\{t \geq S^{\varepsilon} \mid \zeta_{t}>\eta_{t}\right\} .
$$

By left continuity of $\zeta$, we then have $T^{\varepsilon}>S^{\varepsilon}$ on $\left\{S^{\varepsilon}<+\infty\right\}$. Moreover, $S^{\varepsilon}$ is a point of increase for $\eta$ and by assumption on $\eta$ we thus have

$$
X_{S^{\varepsilon}}=Y_{S^{\varepsilon}}=\mathbb{E}\left[\int_{\left(S^{\varepsilon}, T^{\varepsilon}\right]} \eta_{t} \mu(d t) \mid \mathscr{F}_{S^{\varepsilon}}\right]+\mathbb{E}\left[\int_{\left(T^{\varepsilon},+\infty\right]} \eta_{t} \mu(d t) \mid \mathscr{F}_{S^{\varepsilon}}\right] .
$$

By definition of $T^{\varepsilon}$, the first of these conditional expectations is strictly larger than $\mathbb{E}\left[\int_{\left(S^{\varepsilon}, T^{\varepsilon}\right]} \zeta_{t} \mu(d t) \mid \mathscr{F}_{S^{\varepsilon}}\right]$ on $\left\{T^{\varepsilon}>S^{\varepsilon}\right\} \supset\left\{S^{\varepsilon}<+\infty\right\}$. The second conditional expectation equals $\mathbb{E}\left[Y_{T^{\varepsilon}} \mid \mathscr{F}_{S^{\varepsilon}}\right]$ by definition of $Y$, and is thus at least as large as $\mathbb{E}\left[X_{T^{\varepsilon}} \mid \mathscr{F}_{S^{\varepsilon}}\right]$ since $Y$ dominates $X$ by assumption. Hence, on $\left\{S^{\varepsilon}<+\infty\right\}$ we obtain the apparent contradiction that almost surely

$$
\begin{aligned}
X_{S^{\varepsilon}} & >\mathbb{E}\left[\int_{\left(S^{\varepsilon}, T^{\varepsilon}\right]} \zeta_{t} \mu(d t) \mid \mathscr{F}_{S^{\varepsilon}}\right]+\mathbb{E}\left[X_{T^{\varepsilon}} \mid \mathscr{F}_{S^{\varepsilon}}\right] \\
& =\mathbb{E}\left[\int_{\left(S^{\varepsilon}, T^{\varepsilon}\right]} \zeta_{t} \mu(d t) \mid \mathscr{F}_{S^{\varepsilon}}\right]+\mathbb{E}\left[Z_{T^{\varepsilon}} \mid \mathscr{F}_{S^{\varepsilon}}\right] \\
& =Z_{S^{\varepsilon}} \geq X_{S^{\varepsilon}}
\end{aligned}
$$

where for the first equality we used $Z_{T^{\varepsilon}}=X_{T^{\varepsilon}}$ a.s. This equation holds true trivially on $\left\{T^{\varepsilon}=+\infty\right\}$ as $X_{+\infty}=0=Z_{+\infty}$ by assumption, and also on $\left\{T^{\varepsilon}<+\infty\right\}$ since $T^{\varepsilon}$ is a point of increase for $\zeta$ on this set. Clearly, the above contradiction can only be avoided if $\mathbb{P}\left[S^{\varepsilon}<+\infty\right]=0$, i.e., if $\eta \leq \zeta+\varepsilon$ on $[0,+\infty)$ almost surely. Since $\varepsilon$ was arbitrary, this entails $\eta \leq \zeta$ on $[0,+\infty) \mathbb{P}$-a.s. Reversing the roles of $\eta$ and $\zeta$ in the above argument yields the converse inequality, and this proves that $Y=Z$ as claimed.

(ii) By our integrability assumption on the progressively measurable process $\xi$ which occurs in the representation (4), the process $\eta_{t}=\sup _{v \in[0, t)} \xi_{v}(t \in(0,+\infty])$ is $\mathbb{P} \otimes \mu$-integrable and the associated process $Y$ with $(9)$ is of class $(D)$. To verify that $Y$ has the desired properties, it only remains to show that $Y_{T}=X_{T}$ for any point of increase $T \in \mathscr{T}$ of $\eta$. So assume that $\eta_{T}<\eta_{t}$ for any $t \in(T,+\infty]$, $\mathbb{P}$-almost surely. Recalling the definition of $\eta$, this entails for $t \downarrow T$ that

$$
\sup _{v \in[0, T)} \xi_{v}=\eta_{T} \leq \eta_{T+} \leq \limsup _{t \searrow T} \xi_{t}=\xi_{T} \mathbb{P}-\text { a.s. }
$$


where the last equality follows by upper-right continuity of $\xi$. Hence, $\eta_{t}=$ $\sup _{v \in[0, t)} \xi_{v}=\sup _{v \in[T, t)} \xi_{v}$ for any $t \in(T,+\infty]$ almost surely and so we have in fact

$$
Y_{T}=\mathbb{E}\left[\int_{(T,+\infty]} \eta_{t} \mu(d t) \mid \mathscr{F}_{T}\right]=\mathbb{E}\left[\int_{(T,+\infty]} \sup _{v \in[T, t)} \xi_{v} \mu(d t) \mid \mathscr{F}_{T}\right]=X_{T}
$$

where the last equality follows from representation (4).

(iii) Since the right continuous modification of $\eta$ is an increasing, adapted process, we can easily represent $Y$ as required by Theorem 1.3 .

$$
Y_{T}=\mathbb{E}\left[\int_{(T,+\infty]} \sup _{v \in[T, t)} \eta_{v+} \mu(d t) \mid \mathscr{F}_{T}\right] \quad(T \in \mathscr{T}) .
$$

Hence, the stopping times maximizing $\mathbb{E} Y_{T}$ over $T \in \mathscr{T}$ are exactly those stopping times $T^{*}$ such that

$$
\underline{T} \leq T^{*} \leq \bar{T} \mathbb{P}-\text { a.s. } \quad \text { and } \sup _{v \in\left[0, T^{*}\right]} \eta_{v+}=\eta_{T^{*}+} \mathbb{P} \text {-a.s. on }\left\{T^{*}<+\infty\right\}
$$

where

$$
\underline{T} \triangleq \inf \left\{t \in(0,+\infty] \mid \eta_{t+} \geq 0\right\}=\inf \left\{t \in(0,+\infty] \mid \eta_{t} \geq 0\right\}
$$

and

$$
\bar{T} \triangleq \inf \left\{t \in(0,+\infty] \mid \eta_{t+}>0\right\}=\inf \left\{t \in(0,+\infty] \mid \eta_{t}>0\right\} .
$$

By monotonicity of $\eta$, the second condition in 10 is actually redundant, and so a stopping time $T^{*}$ is optimal for $Y$ iff

$$
\underline{T} \leq T^{*} \leq \bar{T} \mathbb{P}-\text { a.s. }
$$

In particular, both $\underline{T}$ and $\bar{T}$ are optimal stopping times for $Y$. In addition, $\bar{T}$ is a time of increase for $\eta$. Thus, $X_{\bar{T}}=Y_{\bar{T}} \mathbb{P}$-a.s. and

$$
\max _{T \in \mathscr{T}} \mathbb{E} X_{T} \geq \mathbb{E} X_{\bar{T}}=\mathbb{E} Y_{\bar{T}}=\max _{T \in \mathscr{T}} \mathbb{E} Y_{T} .
$$

But since $Y \geq X$ by assumption, we have in fact equality everywhere in the above expression, and so the values of the optimal stopping problems for $X$ and $Y$ coincide, and we obtain that any optimal stopping time $T^{*}$ for $X$ must satisfy $X_{T^{*}}=Y_{T^{*}}$ and it must also be an optimal stopping time for $Y$, i.e., satisfy $\underline{T} \leq T^{*} \leq \bar{T}$ almost surely. Conversely, an optimal stopping time $T^{*}$ for $Y$ which in addition satisfies $X_{T^{*}}=Y_{T^{*}}$ almost surely will also be optimal for $X$. 
Let us finally prove that $\underline{T}$ is also an optimal stopping time for $X$. Since $\underline{T}$ is known to be optimal for $Y$ it suffices by the above criterion to verify that $X_{\underline{T}}=\breve{X}_{\underline{T}}$ almost surely. By definition of $Y$ this identity holds true trivially on the set where $\eta$ crosses the zero level by a jump at time $\underline{T}$, since then $\underline{T}$ is obviously a point of increase for $\eta$. To prove this identity also on the complementary set, consider the increasing sequence of stopping times

$$
T^{n} \triangleq \inf \left\{t \in[0, \underline{T}) \mid \eta_{t}>-1 / n\right\} \quad(n=1,2, \ldots) .
$$

By definition, each $T^{n}$ is a time of increase for $\eta$, and thus $X_{T^{n}}=Y_{T^{n}}$ holds true almost surely by the properties of $Y$. Moreover, the stopping times $T^{n}$ increase to the restriction $\underline{T}^{\prime}$ of $\underline{T}$ to the set where $\eta$ continuously approaches its zero level:

$$
T^{n} \rightarrow \underline{T}^{\prime}= \begin{cases}\underline{T} & \text { on }\left\{\eta_{\underline{T}-}=0\right\} \\ +\infty & \text { on }\left\{\eta_{\underline{T}-}<0\right\}\end{cases}
$$

Indeed, on $\left\{\underline{T}^{\prime}<+\infty\right\}$, the stopping times $T^{n}$ converge to $\underline{T}^{\prime}$ strictly from below. It follows that

$$
\mathbb{E} X_{T^{n}}=\mathbb{E} Y_{T^{n}}=\mathbb{E} \int_{\left(T^{n},+\infty\right]} \eta_{t} \mu(d t) \rightarrow \mathbb{E}\left[\int_{\left[\underline{T}^{\prime},+\infty\right]} \eta_{t} \mu(d t) ; \underline{T^{\prime}}<+\infty\right]=\mathbb{E} Y_{\underline{T}^{\prime}}
$$

where the last identity holds true because $\eta_{\underline{T}^{\prime}}=0$ on $\left\{\underline{T^{\prime}}<+\infty\right\}$.

Since $Y$ dominates $X$ the right side of the above expression is $\geq \mathbb{E} X_{\underline{T}^{\prime}}$. On the other hand, in the limit $n \uparrow+\infty$, its left side is not larger than $\mathbb{E} X_{\underline{T}^{\prime}}$ since $X$ is upper semicontinuous in expectation. Hence, we must have $\mathbb{E} Y_{\underline{T}^{\prime}}=\mathbb{E} X_{\underline{T}^{\prime}}$ which implies that in fact $Y_{\underline{T}^{\prime}}=X_{\underline{T}^{\prime}}$ almost surely, as we wanted to show.

Remark 1.7 Parts (i) and (ii) of the above theorem can be seen as a uniqueness and existence result for a variant of Skorohod's obstacle problem, if the optional process $X$ is viewed as a randomly fluctuating obstacle on the real line. With this interpretation, we can consider the set of all class (D) processes $Y$ which never fall below the obstacle $X$ and which follow a backward semimartingale dynamics of the form

$$
d Y_{t}=-\eta_{t} d \mu((0, t])+d M_{t} \quad \text { and } \quad Y_{+\infty}=0
$$


for some uniformly integrable martingale $M$ and for some adapted, left continuous, and non-decreasing process $\eta \in L^{1}(\mathbb{P} \otimes \mu)$. Rewriting the above dynamics in integral form and taking conditional expectations, we see that any such $Y$ takes the form

$$
Y_{T}=\mathbb{E}\left[\int_{(T,+\infty]} \eta_{t} \mu(d t) \mid \mathscr{F}_{T}\right] \quad(T \in \mathscr{T}) .
$$

Clearly, there will be many non-decreasing processes $\eta$ which control the corresponding process $Y$ in such a way that it never falls below the obstacle $X$. However, one could ask whether there is any such process $\eta$ which only increases when necessary, i.e., when its associated process $Y$ actually hits the obstacle $X$, and whether such a minimal process $\eta$ is uniquely determined. The results of Bank and El Karoui (2002) as stated in parts (i) and (ii) of Corollary 1.5 give affirmative answers to both questions under general conditions.

\subsubsection{Universal exercise signals for American options}

In Section 1.1.1, we have seen how the optimal stopping problem for American options can be solved by using Snell envelopes. In particular, an American put option with strike $k$ is optimally exercised, for instance, at time

$$
T^{k} \triangleq \inf \left\{t \in[0, \hat{T}] \mid U_{t}^{k}=e^{-r t}\left(k-P_{t}\right)^{+}\right\},
$$

where $U_{t}^{k}$ is the Snell envelope of the discounted payoff process $\left(e^{-r t}\left(k-P_{t}\right)^{+}\right)_{t \in[0, \hat{T}]}$. Clearly, this construction of the optimal exercise rule is specific for the strike $k$ considered. In practice, however, American put options are traded for a whole variety of different strike prices, and computing all relevant Snell envelopes may turn into a tedious task. Thus, it would be convenient to have a single reference process which allows one to determine optimal exercise times simultaneously for any possible strike $k$. In fact, it is possible to construct such a universal signal using the stochastic representation approach to optimal stopping developed in the preceding section:

Theorem 1.8 Assume that the discounted value process $\left(e^{-r t} P_{t}\right)_{t \in[0, \hat{T}]}$ is an optional process of class (D) which is lower-semicontinuous in expectation.

Then this process admits a unique representation

$$
e^{-r T} P_{T}=\mathbb{E}\left[\int_{(T, \hat{T}]} r e^{-r t} \inf _{v \in[T, t)} K_{v} d t+e^{-r \hat{T}} \inf _{v \in[T, \hat{T}]} K_{v} \mid \mathscr{F}_{T}\right] \quad(T \in \mathscr{T}([0, \hat{T}]))
$$

for some progressively measurable process $K=\left(K_{t}\right)_{t \in[0, \hat{T}]}$ with lower-right continuous paths such that

$$
r e^{-r t} \inf _{v \in[T, t)} K_{v} 1_{(T, \hat{T}]}(t) \in L^{1}(\mathbb{P} \otimes d t) \quad \text { and } \quad e^{-r \hat{T}} \inf _{v \in[T, \hat{T}]} K_{v} \in L^{1}(\mathbb{P})
$$


for all $T \in \mathscr{T}([0, \hat{T}])$.

The process $K$ provides a universal exercise signal for all American put options on the underlying process $P$ in the sense that for any strike $k \geq 0$ the level passage times

$$
\underline{T}^{k} \triangleq \inf \left\{t \in[0, \hat{T}] \mid K_{t} \leq k\right\} \quad \text { and } \quad \bar{T}^{k} \triangleq \inf \left\{t \in[0, \hat{T}] \mid K_{t}<k\right\}
$$

provide the smallest and the largest solution, respectively, of the optimal stopping problem

$$
\max _{T \in \mathscr{T}([0, \hat{T}] \cup\{+\infty\})} \mathbb{E}\left[e^{-r T}\left(k-P_{T}\right) ; T \leq \hat{T}\right] .
$$

In fact, a stopping time $T^{k} \in \mathscr{T}([0, \hat{T}] \cup\{+\infty\})$ is optimal in this sense iff

$$
\underline{T}^{k} \leq T^{k} \leq \bar{T}^{k} \quad \mathbb{P} \text {-a.s. and } \inf _{v \in\left[0, T^{k}\right]} K_{v}=K_{T^{k}} \mathbb{P}-\text { a.s. on }\left\{T^{k} \leq \hat{T}\right\} .
$$

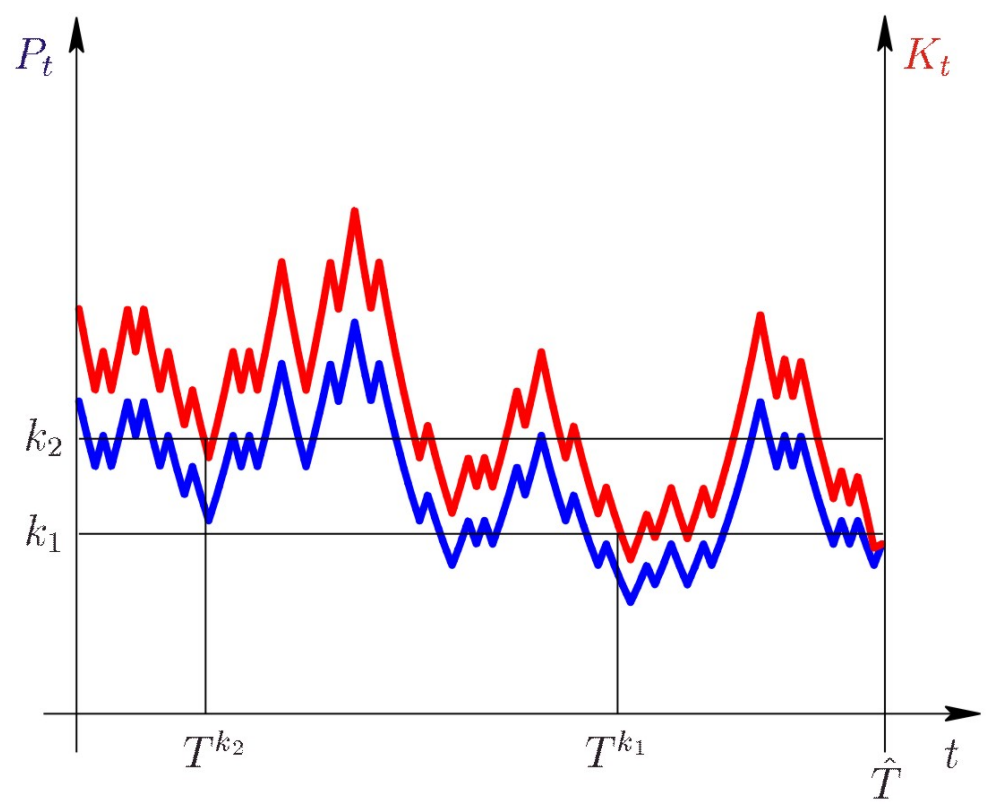

Figure 1: Universal exercise signal $K$ (red line) for an underlying $P$ (blue line), and optimal stopping times $T^{k_{1}}, T^{k_{2}}$ for two different strikes $k_{1}<k_{2}$ (black lines).

Remark 1.9 The preceding theorem is inspired by the results of El Karoui and Karatzas (1995). Their equation (1.4) states the following representation for the early exercise premium of an American put:

$$
\begin{aligned}
& \underset{T \in \mathscr{T}([S, \hat{T}])}{\operatorname{essup}} \mathbb{E}\left[e^{-r(T-S)}\left(k-P_{T}\right)^{+} \mid \mathscr{F}_{S}\right]-\mathbb{E}\left[e^{-r(\hat{T}-S)}\left(k-P_{\hat{T}}\right)^{+} \mid \mathscr{F}_{S}\right] \\
& =\mathbb{E}\left[\int_{(S, T]} r e^{-r(t-S)}\left(k-\inf _{v \in[S, t)} K_{v}\right)^{+} d t+e^{-r(\hat{T}-S)}\left(k \wedge P_{\hat{T}}-\inf _{v \in[S, \hat{T})} K_{v}\right)^{+} \mid \mathscr{F}_{S}\right] .
\end{aligned}
$$


This representation involves the same process $K$ as considered in our Theorem 1.8. In fact, their formula (5.4), which in our notation reads

$$
\begin{aligned}
\lim _{k \uparrow+\infty} & \left\{k-\operatorname{ess}_{T \in \mathscr{T}([S, \hat{T}])} \mathbb{E}\left[e^{-r(T-S)}\left(k-P_{T}\right)^{+} \mid \mathscr{F}_{S}\right]\right\} \\
= & \mathbb{E}\left[\int_{(T, \hat{T}]} r e^{-r(t-S)} \inf _{v \in[T, t)} K_{v} d t+e^{-r(\hat{T}-S)} \inf _{v \in[T, \hat{T}]} K_{v} \mid \mathscr{F}_{S}\right],
\end{aligned}
$$

turns out to be identical with our equation (11) after noting that the limit on the left side coincides with the value of the underlying:

$$
\lim _{k \uparrow+\infty}\left\{k-\operatorname{ess~sup}_{T \in \mathscr{T}([S, \hat{T}])} \mathbb{E}\left[e^{-r(T-S)}\left(k-P_{T}\right)^{+} \mid \mathscr{F}_{S}\right]\right\}=P_{S} \quad \mathbb{P} \text {-a.s. for all } S \in \mathscr{T}([0, \hat{T}]) .
$$

While we use the representation property (11) in order to define the process $K$, El Karoui and Karatzas introduce this process by a Gittins index principle: Their equation (1.3), which in our notation reads

$$
K_{S}=\inf \left\{k>0 \mid \operatorname{essips}_{T \in \mathscr{T}([S, \hat{T}])} \mathbb{E}\left[e^{-r(T-S)}\left(k-P_{T}\right)^{+} \mid \mathscr{F}_{S}\right]=k-P_{S}\right\} \quad(S \in \mathscr{T}([0, \hat{T}]),
$$

defines $K_{S}$ as the minimal strike for which the corresponding American put is optimally exercised immediately at time $S$. Thus, the process $K$ is specified in terms of Snell envelopes. In contrast, our approach defines $K$ directly as the solution to the representation problem (11), and it emphasizes the role of $K$ as a universal exercise signal. In homogeneous models, it is often possible to solve the representation problem directly, without first solving some optimization problem. This shortcut will be illustrated in Section 3 where we shall derive some explicit solutions.

\section{PROOF :}

(i) Existence of a representation for the discounted value process $\left(e^{-r t} P_{t}\right)_{t \in[0, \hat{T}]}$ as in (11) follows from a general representation theorem which will be proved in the next section; confer Corollary 2.4.

(ii) For any strike $k \geq 0$, let us consider the optional payoff process $X^{k}$ defined by

$$
X_{t}^{k} \triangleq e^{-r t}\left(k-P_{t \wedge \hat{T}}\right) \quad(t \in[0,+\infty]) .
$$

We claim that the stopping times $T^{k}$ maximizing $\mathbb{E} X_{T}^{k}$ over $T \in \mathscr{T}$ are exactly those stopping times which maximize $\mathbb{E}\left[e^{-r T}\left(k-P_{T}\right) ; T \leq \hat{T}\right]$ over $T \in$ 
$\mathscr{T}([0, \hat{T}] \cup\{+\infty\})$. In fact, a stopping time $T^{k} \in \mathscr{T}$ maximizing $\mathbb{E} X_{T}^{k}$ will actually take values in $[0, \hat{T}] \cup\{+\infty\}$ almost surely because interest rates $r$ are strictly positive by assumption. Hence, we have

$$
\begin{aligned}
\max _{T \in \mathscr{T}} \mathbb{E} X_{T}^{k} & =\mathbb{E} X_{T^{k}}^{k}=\mathbb{E}\left[e^{-r T^{k}}\left(k-P_{T^{k}}\right) ; T^{k} \leq \hat{T}\right] \\
& \leq \max _{T \in \mathscr{T}([0, \hat{T}] \cup\{+\infty\})} \mathbb{E}\left[e^{-r T}\left(k-P_{T}\right) ; T \leq \hat{T}\right] .
\end{aligned}
$$

On the other hand, we have

$$
\mathbb{E}\left[e^{-r T}\left(k-P_{T}\right) ; T \leq \hat{T}\right]=\mathbb{E} X_{T}^{k}
$$

for any $T \in \mathscr{T}([0, \hat{T}] \cup\{+\infty\})$, again by strict positivity of interest rates. As a consequence, the last max coincides with the first max and both lead to the same set of maximizers.

(iii) We wish to apply Theorem 1.3 in order to solve the optimal stopping problem for $X^{k}(k \geq 0)$ as defined in step (ii) of the present proof. To this end, let us construct a representation

$$
X_{T}^{k}=\mathbb{E}\left[\int_{(T,+\infty]} \sup _{v \in[T, t)} \xi_{v}^{k} \mu(d t) \mid \mathscr{F}_{T}\right] \quad(T \in \mathscr{T})
$$

as required by this theorem. In fact, let

$$
\xi_{t}^{k} \triangleq k-K_{t \wedge \hat{T}} \quad(t \in[0,+\infty))
$$

and put $\mu(d t) \triangleq r e^{-r t} d t$. Then $\xi^{k}$ is obviously a progressively measurable process with upper-right continuous paths and we have for $T \in \mathscr{T}$ :

$$
\begin{aligned}
& \mathbb{E}\left[\int_{(T,+\infty]} \sup _{v \in[T, t)} \xi_{v}^{k} \mu(d t) \mid \mathscr{F}_{T}\right] \\
& =\mathbb{E}\left[\int_{(T,+\infty]} r e^{-r t}\left(k-\inf _{v \in[T, t)} K_{v \wedge \hat{T}}\right) d t \mid \mathscr{F}_{T}\right] \\
& =e^{-r T} k-\mathbb{E}\left[\int_{(T \wedge \hat{T}, \hat{T}]} r e^{-r t} \inf _{v \in[T \wedge \hat{T}, t)} K_{v}+\int_{(T \vee \hat{T},+\infty]} r e^{-r t} \inf _{v \in[T \wedge \hat{T}, \hat{T}]} K_{v} d t \mid \mathscr{F}_{T}\right] \\
& =e^{-r T} k-\mathbb{E}\left[\int_{(T \wedge \hat{T}, \hat{T}]} r e^{-r t} \inf _{v \in[T \wedge \hat{T}, t)} K_{v}+e^{-r T \vee \hat{T}} \inf _{v \in[T \wedge \hat{T}, \hat{T}]} K_{v} \mid \mathscr{F}_{T}\right] \\
& =e^{-r T}\left(k-P_{T \wedge \hat{T}}\right) .
\end{aligned}
$$

Here, the last identity holds true on $\{T \leq \hat{T}\}$ because of the representation property 11 of $K$, and also on the complementary event $\{T>\hat{T}\}$, since on this set $\inf _{v \in[T \wedge \hat{T}, \hat{T}]} K_{v}=K_{\hat{T}}=P_{\hat{T}}$, again by (11). 
(iv) Applying Theorem 1.3 to $X=X^{k}$, we obtain that $T^{k} \in \mathscr{T}$ maximizes $\mathbb{E} X_{T}^{k}$ over all $T \in \mathscr{T}$ iff

$$
\underline{T}^{k} \leq T^{k} \leq \bar{T}^{k} \mathbb{P} \text {-a.s. and } \sup _{v \in\left[0, T^{k}\right]} \xi_{v}^{k}=\xi_{T^{k}}^{k} \mathbb{P} \text {-a.s. on }\left\{T^{k}<+\infty\right\},
$$

where $\underline{T}^{k} \triangleq \inf \left\{t \geq 0 \mid \xi_{t}^{k} \geq 0\right.$ and $\bar{T}^{k}=\inf \left\{t \geq 0 \mid \xi_{t}^{k}>0\right\}$. Recalling the definition of $\xi^{k}$ and that $\left\{T^{k}<+\infty\right\}=\left\{T^{k} \leq \hat{T}\right\}$ for any optimal stopping time for $X^{k}$ by (ii), we see that this condition is actually equivalent to the criterion in $(12)$.

Let us now apply Theorem 1.8 to the usual put option profile $\left(e^{-r t}(k-P)^{+}\right)_{t \in[0, \hat{T}]}$.

Corollary 1.10 The universal exercise signal $K=\left(K_{t}\right)_{t \geq 0}$ characterized by (11) satisfies $K_{T} \geq P_{T}$ for all $T \in \mathscr{T}([0, \hat{T}])$ almost surely. In particular, the restriction $T^{k} \wedge \hat{T}$ of any optimal stopping time $T^{k}$ as characterized in Theorem 1.8 also maximizes $\mathbb{E} e^{-r T}\left(k-P_{T}\right)^{+}$among all stopping times $T \in \mathscr{T}([0, \hat{T}])$.

Proof : For any $T \in \mathscr{T}([0, \hat{T}])$, the representation 11$]$ implies

$$
\begin{aligned}
e^{-r T} P_{T} & =\mathbb{E}\left[\int_{(T, \hat{T}]} r e^{-r t} \inf _{v \in[T, t)} K_{v} d t+e^{-r \hat{T}} \inf _{v \in[T, \hat{T}]} K_{v} \mid \mathscr{F}_{T}\right] \\
& \leq \mathbb{E}\left[\int_{(T, \hat{T}]} r e^{-r t} K_{T} d t+e^{-r \hat{T}} K_{T} \mid \mathscr{F}_{T}\right] \\
& =e^{-r T} K_{T}
\end{aligned}
$$

almost surely. In particular, $P_{T^{k}} \leq K_{T^{k}} \leq k$ almost surely on $\left\{T^{k} \leq \hat{T}\right\}$ for any optimal stopping time $T^{k}$ as in Theorem 1.8 . Thus,

$$
\mathbb{E}\left[e^{-r T^{k}}\left(k-P_{T^{k}}\right) ; T^{k} \leq \hat{T}\right]=\mathbb{E}\left[e^{-r T^{k} \wedge T}\left(k-P_{T^{k} \wedge \hat{T}}\right)^{+}\right]
$$

and so $T^{k} \wedge \hat{T}$ maximizes $\mathbb{E} e^{-r T}\left(k-P_{T}\right)^{+}$over $T \in \mathscr{T}([0, \hat{T}])$.

Using the same arguments as in the proof of Theorem 1.11, we can also construct universal exercise signals for American call options:

Theorem 1.11 Assume the discounted value process $\left(e^{-r t} P_{t}\right)_{t \in[0, \hat{T}]}$ is an optional process of class $(D)$ which is upper-semicontinuous in expectation. Then this process admits a unique representation

$$
e^{-r T} P_{T}=\mathbb{E}\left[\int_{(T, \hat{T}]} r e^{-r t} \sup _{v \in[T, t)} K_{v} d t+e^{-r \hat{T}} \sup _{v \in[T, \hat{T}]} K_{v} \mid \mathscr{F}_{T}\right] \quad(T \in \mathscr{T}([0, \hat{T}]))
$$


for some progressively measurable process $K$ with upper-right continuous paths and

$$
r e^{-r t} \sup _{v \in[T, t)} K_{s} 1_{(T, \hat{T}]}(t) \in L^{1}(\mathbb{P} \otimes d t) \quad \text { and } \quad e^{-r \hat{T}} \sup _{v \in[T, \hat{T}]} K_{v} \in L^{1}(\mathbb{P})
$$

for all $T \in \mathscr{T}([0, \hat{T}])$.

This process $K$ provides a universal exercise signal for all American call options with underlying $P$ in the sense that for any strike $k \geq 0$ the level passage times

$$
\underline{T}^{k} \triangleq \inf \left\{t \in[0, \hat{T}] \mid K_{t} \geq k\right\} \quad \text { and } \quad \bar{T}^{k} \triangleq \inf \left\{t \in[0, \hat{T}] \mid K_{t}>k\right\}
$$

provide the smallest and the largest solution, respectively, of the optimal stopping problem

$$
\max _{T \in \mathscr{T}([0, \hat{T}] \cup\{+\infty\})} \mathbb{E}\left[e^{-r T}\left(P_{T}-k\right) ; T \leq \hat{T}\right] .
$$

In fact, a stopping time $T^{k}$ is optimal in this sense iff

$$
\underline{T}^{k} \leq T^{k} \leq \bar{T}^{k} \mathbb{P}-\text { a.s. and } \sup _{v \in\left[0, T^{k}\right]} K_{v}=K_{T^{k}} \mathbb{P}-\text { a.s. on }\left\{T^{k}<+\infty\right\} .
$$

The preceding theorem solves the optimal stopping problem of American calls under a general probability measure $\mathbb{P}$. For example, $\mathbb{P}$ could specify the probabilistic model used by the buyer of the option. From the point of view of the option seller and in the context of a complete financial market model, however, the problem should be formulated in terms of the equivalent martingale $\mathbb{P}^{*}$. In this case, the payoff process of the call option is a submartingale, and the optimal stopping problem is clearly solved by the simple rule: "Always stop at the terminal time $\hat{T}$ ". In the preceding theorem, this is reflected by the fact that the process $K$ takes the simple form $K_{t}=0$ for $t \in[0, \hat{T})$ and $K_{\hat{T}}=P_{\hat{T}}$.

Remark 1.12 The results of this section also apply when interest rates $r=\left(r_{t}\right)_{0 \leq t \leq \hat{T}}$ follow a progressively measurable process, provided this process is integrable and strictly positive. For instance, the representation (11) then takes the form

$$
e^{-\int_{0}^{T} r_{s} d s} P_{T}=\mathbb{E}\left[\int_{(T, \hat{T}]} r_{t} e^{-\int_{0}^{t} r_{s} d s} \inf _{v \in[T, t)} K_{v} d t+e^{-\int_{0}^{\hat{T}} r_{s} d s} \inf _{v \in[T, \hat{T}]} K_{v} \mid \mathscr{F}_{T}\right]
$$

for $T \in \mathscr{T}([0, \hat{T}])$. 


\subsection{Optimal consumption plans}

In this section, we discuss a singular control problem arising in the microeconomic theory of intertemporal consumption choice. We shall show how this problem can be reduced to a stochastic representation problem of the same type as in the previous section.

Consider an economic agent who makes a choice among different consumption plans. A consumption pattern is described as a positive measure on the time axis $[0,+\infty)$ or, in a cumulative way, by the corresponding distribution function. Thus, a consumption plan which is contingent on scenarios is specified by an element in the set

$$
\mathscr{C} \triangleq\{C \geq 0 \mid C \text { is a right continuous, increasing and adapted process }\} \text {. }
$$

Given some initial wealth $w>0$, the agent's budget set is of the form

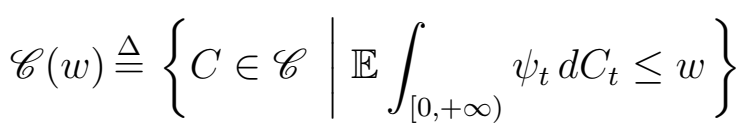

where $\psi=\left(\psi_{t}\right)_{t \in[0,+\infty)}>0$ is a given optional price deflator.

Remark 1.13 Consider a financial market model specified by an $\mathbb{R}^{d}$-valued semimartingale $\left(P_{t}\right)_{t \in[0,+\infty)}$ of asset prices and an optional process $\left(r_{t}\right)_{t \in[0,+\infty)}$ of interest rates. Absence of arbitrage opportunities can be guaranteed by the existence of an equivalent local martingale measures $\mathbb{P}^{*} \approx \mathbb{P} ;$ cf. Delbaen and Schachermayer (1994). An initial capital $V_{0}$ is sufficient to implement a given consumption plan $C \in \mathscr{C}$ if there is a trading strategy, given by a d-dimensional predictable process $\left(\theta_{t}\right)_{t \in[0,+\infty)}$, such that the resulting wealth process

$$
V_{t}=V_{0}+\int_{0}^{t} \theta_{s} d P_{s}+\int_{0}^{t}\left(V_{s}-\theta_{s} P_{s}\right) r_{s} d s-C_{t} \quad(t \in[0,+\infty))
$$

remains nonnegative. Thus, the cost of implementing the consumption plan $C$ should be defined as the smallest such value $V_{0}$. Dually, this cost can be computed as

$$
\sup _{\mathbb{P}^{*} \in \mathscr{P} *} \mathbb{E}^{*} \int_{0}^{+\infty} e^{-\int_{0}^{t} r_{s} d s} d C_{s}
$$

where $\mathscr{P}^{*}$ denotes the class of all equivalent local martingale measures; this follows from a theorem on optional decompositions which was proved in increasing generality by El Karoui and Quenez (1995), Kramkov (1996), and Föllmer and Kabanov (1998). In the case of a complete financial market model, the equivalent martingale measure $\mathbb{P}^{*}$ is unique, and the cost takes the form appearing in (14), with

$$
\left.\psi_{t} \triangleq e^{-\int_{0}^{t} r_{s} d s} \frac{d \mathbb{P}^{*}}{d \mathbb{P}^{*}}\right|_{\mathscr{F}_{t}} \quad(t \in[0,+\infty))
$$


The choice of a specific consumption plan $C \in \mathscr{C}(w)$ will depend on the agent's preferences. A standard approach in the Finance literature consists in restricting attention to the set $\mathscr{C}_{\text {ac }}$ of absolutely continuous consumption plans

$$
C_{t}=\int_{0}^{t} c_{s} d s \quad(t \in[0,+\infty))
$$

where the progressively measurable process $c=\left(c_{t}\right)_{t \in[0,+\infty)} \geq 0$ specifies a rate of consumption. For a time-dependent utility function $u(t,$.$) , the problem of finding the$ best consumption plan $C^{*}$ in $\mathscr{C}(w) \cap \mathscr{C}_{\text {ac }}$ is then formulated in terms of the utility functional

$$
U_{\mathrm{ac}}(C) \triangleq \mathbb{E} \int_{0}^{+\infty} u\left(t, c_{t}\right) d t .
$$

From a mathematical point of view, this is a space-time version of the standard problem of maximizing expected utility under a linear budget constraint, and its solution is straightforward; see, e.g., Karatzas and Shreve (1998).

However, as shown in Hindy, Huang, and Kreps (1992), a utility functional of the time-additive form (15) raises serious objections, both from an economic and a mathematical point of view. Firstly, a reasonable extension of the functional $U_{\mathrm{ac}}$ from $\mathscr{C}_{\mathrm{ac}}$ to $\mathscr{C}$ only works for spatially affine functions $u$. Secondly, such functionals are not robust with respect to small time-shifts in consumption plans, and thus do not capture intertemporal substitution effects. Finally, the price functionals arising in the corresponding equilibrium analysis, viewed as continuous linear functionals on the space $\mathscr{C}_{\text {ac }}$ with respect to an $L^{p}$-norm on consumption rates, fail to have desirable properties such as the existence of an interest rate. For such reasons, Hindy, Huang, and Kreps (1992) introduce utility functionals of the following type.

$$
U(C) \triangleq \mathbb{E} \int_{(0,+\infty]} u\left(t, Y_{t}^{C}\right) \nu(d t) \quad(C \in \mathscr{C}),
$$

where $\nu$ is a nonnegative optional random measure, and where

$$
Y_{t}^{C} \triangleq \eta e^{-\beta t}+\int_{[0, t)} \beta e^{-\beta(t-s)} d C_{s} \quad(t \geq 0)
$$

serves as an index of satisfaction, defined as an exponential average of past consumption. The measure $\nu$ accounts for the agent's time preferences. For fixed $t \geq 0$, the utility function $u(t, y)$ is assumed to be strictly concave and increasing in $y \in[0,+\infty)$ with continuous partial derivative $\partial_{y} u(t, y)$. We assume $\partial_{y} u(t, 0) \equiv+\infty, \partial_{y} u(t,+\infty) \equiv 0$, and $\partial_{y} u(., y) \in L^{1}(\mathbb{P} \otimes \nu)$ for any $y>0$. 
With this choice of preferences, the agent's optimization problem consists in maximizing the concave functional $U$ under a linear constraint:

$$
\text { Maximize } U(C) \text { subject to } C \in \mathscr{C}(w) \text {. }
$$

In Hindy and Huang (1993), this problem is analyzed in a Markovian setting, using the Hamilton-Jacobi-Bellman approach; see also Benth, Karlsen, and Reikvam (2001).

Let us now describe an alternative approach developed in Bank and Riedel (2001) under the natural assumption that

$$
\sup _{C \in \mathscr{C}(w)} U(C)<+\infty \text { for any } w>0 .
$$

This approach can be applied in a general semimartingale setting, and it leads to a stochastic representation problem of the same type as in the previous section. It is based on the following Kuhn-Tucker criterion for optimality of a consumption plan:

Lemma 1.14 A consumption plan $C^{*} \in \mathscr{C}$ is optimal for its cost

$$
w \triangleq \mathbb{E} \int_{[0,+\infty)} \psi_{t} d C_{t}^{*}<+\infty
$$

if it satisfies the first order condition

$$
\nabla U\left(C^{*}\right) \leq \lambda \psi \quad \text {, with equality } \mathbb{P} \otimes d C^{*} \text {-a.e. }
$$

for some Lagrange multiplier $\lambda>0$, where the gradient $\nabla U\left(C^{*}\right)$ is defined as the unique optional process such that

$$
\nabla U\left(C^{*}\right)_{T}=\mathbb{E}\left[\int_{(T,+\infty]} \beta e^{-\beta(t-T)} \partial_{y} u\left(t, Y_{t}^{C^{*}}\right) \nu(d t) \mid \mathscr{F}_{T}\right] \quad \text { for all } \quad T \in \mathscr{T} .
$$

Proof : Let $C^{*}$ be as above and take an arbitrary plan $C \in \mathscr{C}(w)$. By concavity we can estimate

$$
\begin{aligned}
U(C)-U\left(C^{*}\right) & =\mathbb{E} \int_{(0,+\infty]}\left\{u\left(t, Y_{t}^{C}\right)-u\left(t, Y_{t}^{C^{*}}\right)\right\} \nu(d t) \\
& \leq \mathbb{E} \int_{(0,+\infty]} \partial_{y} u\left(t, Y_{t}^{C^{*}}\right)\left\{Y_{t}^{C}-Y_{t}^{C^{*}}\right\} \nu(d t) \\
& =\mathbb{E} \int_{(0,+\infty]} \partial_{y} u\left(t, Y_{t}^{C^{*}}\right)\left\{\int_{[0, t)} \beta e^{-\beta(t-s)}\left(d C_{s}-d C_{s}^{*}\right)\right\} \nu(d t) .
\end{aligned}
$$


Using Fubini's theorem we thus obtain

$$
\begin{aligned}
U(C)-U\left(C^{*}\right) & \leq \mathbb{E} \int_{[0,+\infty)}\left\{\int_{(s,+\infty]} \beta e^{-\beta(t-s)} \partial_{y} u\left(t, Y_{t}^{C^{*}}\right) \nu(d t)\right\}\left(d C_{s}-d C_{s}^{*}\right) \\
& =\mathbb{E} \int_{[0,+\infty)} \nabla U\left(C^{*}\right)_{s}\left(d C_{s}-d C_{s}^{*}\right)
\end{aligned}
$$

where the last equality follows from Théorème 1.33 in Jacod $(1979)$ since $\nabla U\left(C^{*}\right)$ is the optional projection of the $\left\{\int \ldots \nu(d t)\right\}$-term above. Thus, $\nabla U$ serves as a supergradient of $U$, viewed as a concave functional on the budget set $\mathscr{C}(w)$.

Now, we can use the first order condition to arrive at the estimate

$$
U(C)-U\left(C^{*}\right) \leq \lambda \mathbb{E} \int_{[0,+\infty)} \psi_{s}\left(d C_{s}-d C_{s}^{*}\right)
$$

Since $C \in \mathscr{C}(w)$ and as $C^{*}$ exhausts the budget $w$ by assumption, the last expectation is $\leq 0$, and we can conclude $U(C) \leq U\left(C^{*}\right)$ as desired.

Combining the first order condition for optimality with a stochastic representation of the price deflator process, we now can describe the optimal consumption plans:

Theorem 1.15 Assume that for any $\lambda>0$ the discounted price deflator $\left(\lambda e^{-\beta t} \psi_{t} 1_{[0,+\infty)}(t)\right)_{t \in[0,+\infty]}$ admits a representation

$$
\lambda e^{-\beta T} \psi_{T} 1_{\{T<+\infty\}}=\mathbb{E}\left[\int_{(T,+\infty]} \beta e^{-\beta t} \partial_{y} u\left(t, \sup _{v \in[T, t)}\left\{L_{v} e^{\beta(v-t)}\right\}\right) \nu(d t) \mid \mathscr{F}_{T}\right] \quad(T \in \mathscr{T})
$$

for some progressively measurable process $L=\left(L_{t}\right)_{t \geq 0}>0$ with upper-right continuous paths satisfying

$$
\beta e^{-\beta t} \partial_{y} u\left(t, \sup _{v \in[T, t)}\left\{L_{v} e^{\beta(v-t)}\right\}\right) 1_{(T,+\infty]}(t) \in L^{1}(\mathbb{P} \otimes \nu(d t))
$$

for all $T \in \mathscr{T}$.

Then this process $L$ provides a universal consumption signal in the sense that, for any initial level of satisfaction $\eta$, the unique plan $C^{\eta} \in \mathscr{C}$ such that

$$
Y_{t}^{C^{\eta}}=\eta e^{-\beta t} \vee \sup _{v \in[0, t)}\left\{L_{v} e^{\beta(v-t)}\right\} \quad \text { for all } \quad t \in(0,+\infty]
$$

is optimal for its cost $w=\mathbb{E} \int_{[0,+\infty)} \psi_{t} d C_{t}^{\eta}$.

Thus, the optimal consumption plan consists in consuming just enough to ensure that the induced level of satisfaction $Y^{C^{\eta}}$ stays above the signal process $L$ which appears in the representation (16) of the price deflator process $\psi$. This is illustrated in Figure 1.2 . 


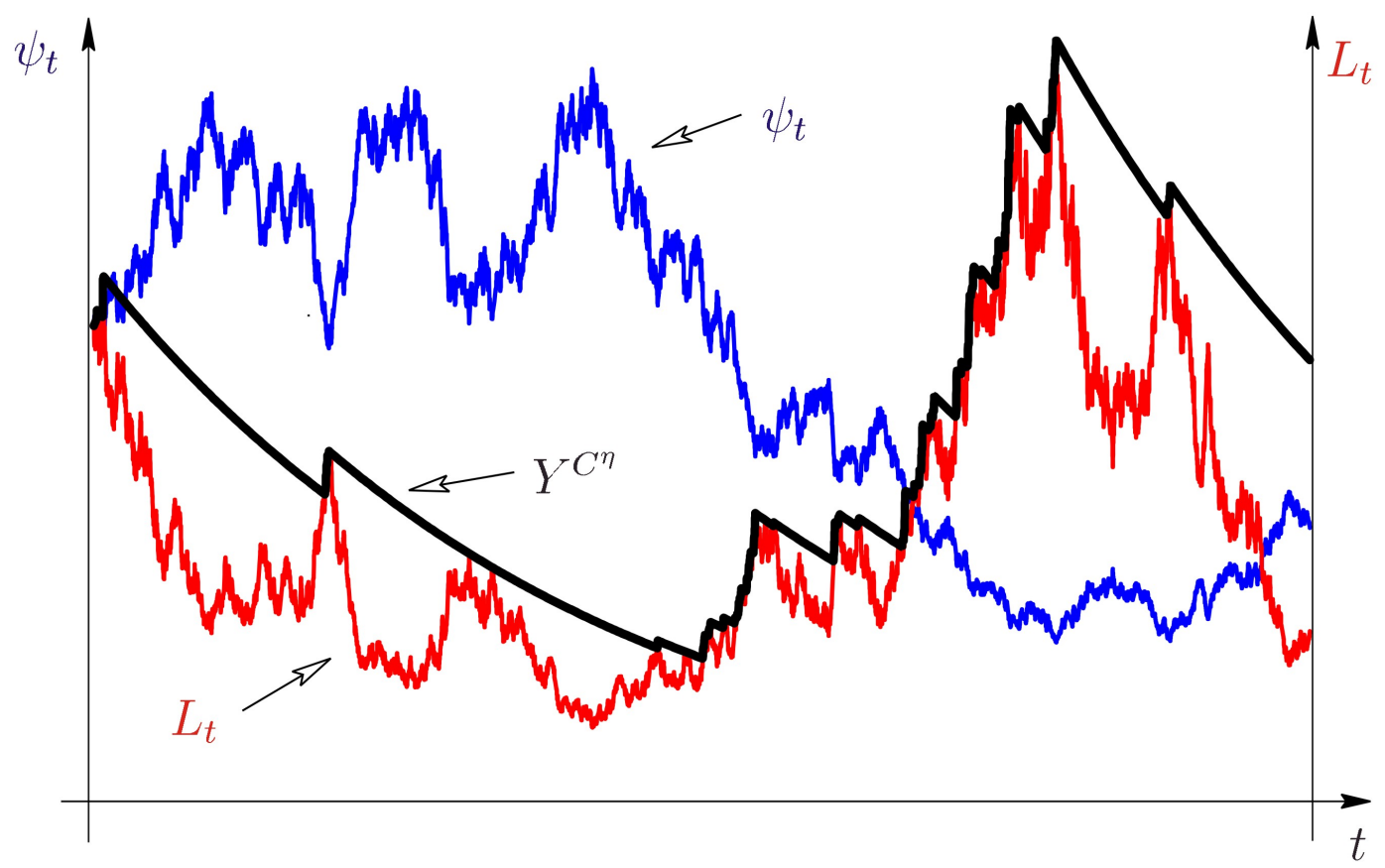

Figure 2: Typical paths for the deflator $\psi$ (blue line), a universal consumption signal $L$ (red line), and the induced level of satisfaction $Y^{C^{\eta}}$ (black line).

Remark 1.16 (i) In case $\mu$ is atomless and has full support almost surely, existence and uniqueness of the process $L$ appearing in (16) follows from a general representation theorem which will be proved in the next section; cf. Corollary 2.4.

(ii) As pointed out in Bank and Riedel (2001), a solution L to the representation problem (16) can be viewed as a minimal level of satisfaction which the agent is willing to accept. Indeed, as shown in Lemma 2.9 of Bank and Riedel (2001), we can represent the process $C^{\eta}$ defined in the preceding theorem in the form

$$
d C_{t}^{\eta}=\frac{e^{-\beta t}}{\beta} d A_{t}^{\eta} \quad(t \in[0,+\infty))
$$

with $A_{t}^{\eta} \triangleq \eta \vee \sup _{v \in[0, t]}\left\{L_{v} e^{\beta v}\right\} \quad(t \in[0,+\infty))$. Hence, if $T \in \mathscr{T}$ is a point of increase for $C^{\eta}$, then it is a point of increase for $A^{\eta}$ and we have

$$
Y_{T+}^{C^{\eta}}=e^{-\beta T} A_{T}^{\eta}=L_{T}
$$

at any such time, while otherwise $Y_{t+}^{C^{\eta}}=e^{-\beta t} A_{t}^{\eta} \geq L_{t}$. 
Proof : We show that the plan $C^{*} \triangleq C^{\eta}$ with the above properties satisfies the first order condition

$$
\nabla U\left(C^{*}\right) \leq \lambda \psi \quad, \text { with equality } \mathbb{P} \otimes d C^{*} \text {-a.e., }
$$

of Lemma 1.14. Indeed, for any $T \in \mathscr{T}$ we have by definition of $C^{*}$ and monotonicity of $\partial_{y} u(t,$.$) :$

$$
\begin{aligned}
\nabla U\left(C^{*}\right)_{T} & =\mathbb{E}\left[\int_{(T,+\infty]} \beta e^{-\beta(t-T)} \partial_{y} u\left(t, Y_{t}^{C^{*}}\right) \nu(d t) \mid \mathscr{F}_{T}\right] \\
& \leq \mathbb{E}\left[\int_{(T,+\infty]} \beta e^{-\beta(t-T)} \partial_{y} u\left(t, \sup _{v \in[T, t)}\left\{L_{v} e^{\beta(v-t)}\right\}\right) \nu(d t) \mid \mathscr{F}_{T}\right]
\end{aligned}
$$

It now follows from the representation property of $L$ that the last conditional expectation is exactly $\lambda \psi_{T} 1_{\{T<+\infty\}}$. Since $T \in \mathscr{T}$ was arbitrary, this implies $\nabla U\left(C^{*}\right) \leq \lambda \psi$. In order to prove that equality holds true $\mathbb{P} \otimes d C^{*}$-a.e. let us consider an arbitrary point of increase for $C^{*}$, i.e., a stopping time $T$ so that $C_{T-}^{*}<C_{t}^{*}$ for all $t \in(T,+\infty)$ almost surely on $\{0<T<+\infty\}$. By definition of $C^{*}$ we obtain

$$
Y_{t}^{C^{*}}=\sup _{v \in[T, t)}\left\{L_{v} e^{\beta(v-t)}\right\} \quad \text { for any } \quad t \in(T,+\infty] \quad \mathbb{P} \text {-a.s. . }
$$

Thus, (18) becomes an equality for any such $T$. It follows that $\nabla U\left(C^{*}\right)=\lambda \psi$ holds true $\mathbb{P} \otimes d C^{*}$-a.e., since the points of increase of $C^{*}$ carry the measure $d C^{*}$.

\subsection{Multi-armed bandits and Gittins indices}

In the multi-armed bandit problem, a gambler faces a slot machine with several arms. All arms yield a payoff of 0 or 1 Euro when pulled, but they may have different payoff probabilities. These probabilities are unknown to the gambler, but playing with the slot machine will allow her to get an increasingly more accurate estimate of each arm's payoff probability. The gambler's aim is to choose a sequence of arms to pull so as to maximize the expected sum of discounted rewards. This choice involves a tradeoff: On the one hand, it seems attractive to pull arms with a currently high estimate of their success probability, on the other hand, one may want to pull other arms to improve the corresponding estimate. In its general form, the multi-armed bandit problem amounts to a dynamic allocation problem where a limited amount of effort is allocated to a number of independent projects, each generating a specific stochastic reward proportional to the effort spent on it. 
Gittins' crucial idea was to introduce a family of simpler benchmark problems and to define a dynamic performance measure - now called the Gittins index - separately for each of the projects in such a way that an optimal schedule can be specified as an indexrule: "Always spent your effort on the projects with currently maximal Gittins index". See Gittins (1979) and Whittle (1980) for the solution in a discrete-time Markovian setting, Karatzas (1984) and Mandelbaum (1987) for an analysis of the diffusion case, and El Karoui and Karatzas (1994) and El Karoui and Karatzas (1997) for a general martingale approach.

To describe the connection between the Gittins index and the representation problems discussed in the preceding sections, let us review the construction of Gittins indices in continuous time. Consider a project whose reward is specified by some rate process $\left(h_{t}\right)_{t \in[0,+\infty)}$. With such a project, El Karoui and Karatzas (1994) associate the family of optimal stopping problems

$$
V_{S}^{m} \triangleq \operatorname{ess} \sup _{T \in \mathscr{T}([S,+\infty])} \mathbb{E}\left[\int_{S}^{T} e^{-\alpha(t-S)} h_{t} d t+m e^{-\alpha(T-S)} \mid \mathscr{F}_{S}\right] \quad(S \in \mathscr{T}, m \geq 0) .
$$

The optimization starts at time $S$, the parameter $m \geq 0$ is interpreted as a rewardupon-stopping, and $\alpha>0$ is a constant discount rate.

Under appropriate conditions, El Karoui and Karatzas (1994) show that the Gittins index $M$ of a project can be described as the minimal reward-upon-stopping such that immediate termination of the project is optimal in the auxiliary stopping problem (19), i.e.:

$$
M_{s}=\inf \left\{m \geq 0 \mid V_{s}^{m}=m\right\} \quad(s \geq 0) .
$$

They also note in their identity (3.7) that $M$ can be related to the reward process $\left(h_{t}\right)$ via

$$
\mathbb{E}\left[\int_{s}^{+\infty} e^{-\alpha t} h_{t} d t \mid \mathscr{F}_{s}\right]=\mathbb{E}\left[\int_{s}^{+\infty} \alpha e^{-\alpha t} \sup _{s \leq v \leq t} M_{v} d t \mid \mathscr{F}_{s}\right] \quad(s \geq 0)
$$

Thus, the Gittins index process $M$ can be viewed as the solution to a representation problem of the form (1). In El Karoui and Karatzas (1994), formula (21) is stated in passing, without making further use of it. Here, we focus on the stochastic representation problem and use it as our starting point. Our main purpose is to emphasize its intrinsic mathematical interest and its unifying role for a number of different applications. In this perspective, formula (20) provides a key to proving existence of a solution to the representation problem in its general form (1), as explained in the next section. 


\section{A stochastic representation theorem}

The previous section has shown how a variety of optimization problems can be reduced to a stochastic representation of a given optional process in terms of running suprema of another process. Let us now discuss the solution of this representation problem from a general point of view.

\subsection{The result and its application}

Let $\mu$ be a nonnegative optional random measure and let $f=f(\omega, t, x): \Omega \times[0,+\infty] \times$ $\mathbb{R} \rightarrow \mathbb{R}$ be a random field with the following properties:

(i) For any $x \in \mathbb{R}$, the mapping $(\omega, t) \mapsto f(\omega, t, x)$ defines a progressively measurable process in $L^{1}(\mathbb{P}(d \omega) \otimes \mu(\omega, d t))$.

(ii) For any $(\omega, t) \in \Omega \times[0,+\infty]$, the mapping $x \mapsto f(\omega, t, x)$ is continuous and strictly decreasing from $+\infty$ to $-\infty$.

Then we can formulate the following general

Representation Problem 2.1 For a given optional process $X=\left(X_{t}\right)_{t \in[0,+\infty]}$ with $X_{+\infty}=0$, construct a progressively measurable process $\xi=\left(\xi_{v}\right)_{v \in[0,+\infty)}$ with upper-right continuous paths such that

$$
f\left(t, \sup _{v \in[T, t)} \xi_{v}\right) 1_{(T,+\infty]}(t) \in L^{1}(\mathbb{P} \otimes \mu(d t))
$$

and

$$
X_{T}=\mathbb{E}\left[\int_{(T,+\infty]} f\left(t, \sup _{v \in[T, t)} \xi_{v}\right) \mu(d t) \mid \mathscr{F}_{T}\right]
$$

for any stopping time $T \in \mathscr{T}$.

This problem is solved by the following result from Bank and El Karoui (2002). Its proof will be discussed in the next section.

Theorem 2.2 If the measure $\mu$ has full support $\operatorname{supp} \mu=[0,+\infty]$ almost surely and $X$ is lower-semicontinuous in expectation, then the solution $\xi$ to representation problem (2.1) is uniquely determined up to optional sections in the sense that

$$
\xi_{S}=\operatorname{essinf}_{T \in \mathscr{T}((S,+\infty])} \Xi_{S, T} \quad \text { for any } \quad S \in \mathscr{T}([0,+\infty))
$$


where $\Xi_{S, T}$ denotes the unique $\mathscr{F}_{S}$-measurable random variable satisfying

$$
\mathbb{E}\left[X_{S}-X_{T} \mid \mathscr{F}_{S}\right]=\mathbb{E}\left[\int_{(S, T]} f\left(t, \Xi_{S, T}\right) \mu(d t) \mid \mathscr{F}_{S}\right] .
$$

If, in addition, $\mu$ almost surely has no atoms, then there actually exists a solution to problem (2.1).

Remark 2.3 If $\mu$ has full support almost surely, we have existence and uniqueness of $\Xi_{S, T} \in L^{0}\left(\mathscr{F}_{S}\right)$ with $(23)$ for any $S \in \mathscr{T}([0,+\infty))$ and any $T \in \mathscr{T}((S,+\infty])$. Indeed, the right side of (23) is then continuous and strictly decreasing in $\Xi=\Xi_{S, T}$ with upper and lower limit $\pm \infty$, respectively. This follows from the corresponding properties of $f=f(\omega, t, x)$ and from the fact that $\mu$ has full support.

As an application of Theorem 2.2, we now can solve all the existence problems arising in our discussion of American put and call options and of optimal consumption plans. This completes the proofs of Theorem 1.8. Theorem 1.11. In the context of Theorem 1.15, this shows that lower-semicontinuity in expectation of the discounted deflator is sufficient for existence of a representation as in (16) if the time-preference measure $\nu$ is atomless and has full support almost surely.

Corollary 2.4 There exist solutions to the representation problems (11), (13), and (16).

\section{ProOF :}

(i) For solving the representation problem (11) which characterizes the universal exercise signal for American put options on $\left(P_{t}\right)_{t \in[0,+\infty)}$, we choose $\mu(d t)=r e^{-r t} d t$ and $f(t, x) \triangleq-x$. Furthermore, we extend $\left(e^{-r t} P_{t}\right)_{t \in[0, \hat{T}]}$ to an optional process $X$ on $[0,+\infty]$ with $X_{+\infty}=0$ :

$$
X_{s} \triangleq e^{-r s} P_{s \wedge \hat{T}}=\int_{(s,+\infty]} r e^{-r t} P_{s \wedge \hat{T}} d t \quad(s \in[0,+\infty)) .
$$

This process is lower-semicontinuous in expectation, due to our assumptions on the process $P$.

Applying Theorem 2.2, we obtain a progressively measurable process $\xi$ with upperright continuous paths such that

$$
X_{T}=\mathbb{E}\left[\int_{(T,+\infty]} f\left(t, \sup _{v \in[T, t)} \xi_{v}\right) \mu(d t) \mid \mathscr{F}_{T}\right]=-\mathbb{E}\left[\int_{(T,+\infty]} r e^{-r t} \sup _{v \in[T, t)} \xi_{v} d t \mid \mathscr{F}_{T}\right]
$$


for all $T \in \mathscr{T}$. Hence, $K \triangleq-\xi$ is lower-right continuous and satisfies

$$
X_{T}=\mathbb{E}\left[\int_{(T,+\infty]} r e^{-r t} \inf _{v \in[T, t)} K_{v} d t \mid \mathscr{F}_{T}\right]
$$

for any $T \in \mathscr{T}$. Comparing this representation with our definition of $X$ on $[\hat{T},+\infty]$, we obtain by uniqueness that $\inf _{T \leq v<t} K_{v}=P_{\hat{T}}$ for any $t>T \geq \hat{T}$. In particular, it follows that $K_{\hat{T}}=P_{\hat{T}}$ by lower-right continuity of $K$. For stopping times $T \in \mathscr{T}([0, \hat{T}])$, expression 24 therefore transforms into

$$
\begin{aligned}
X_{T} & =\mathbb{E}\left[\int_{(T, \hat{T}]} r e^{-r t} \inf _{v \in[T, t)} K_{v} d t+\int_{(\hat{T},+\infty]} r e^{-r t} \inf _{v \in[T, \hat{T})} K_{v} \wedge P_{\hat{T}} d t \mid \mathscr{F}_{T}\right] \\
& =\mathbb{E}\left[\int_{(T, \hat{T}]} r e^{-r t} \inf _{v \in[T, t)} K_{v} d t+e^{-r \hat{T}} \inf _{v \in[T, \hat{T}]} K_{v} \mid \mathscr{F}_{T}\right] .
\end{aligned}
$$

Hence, $K$ solves the representation problem (11).

(ii) The representation problem (13) for American call options can be solved by applying analogous arguments to the process

$$
X_{s} \triangleq-e^{-r s} P_{s \wedge \hat{T}} \quad(s \in[0,+\infty]) .
$$

(iii) For the representation problem (16) which arises in the context of intertemporal consumption choice, we choose $\mu(d t) \triangleq \beta e^{-\beta t} \nu(d t)$,

$$
f(t, x) \triangleq \begin{cases}\partial_{y} u\left(t,-e^{-\beta t} / x\right), & x<0 \\ -x, & x \geq 0\end{cases}
$$

and $X_{t} \triangleq \lambda e^{\beta t} \psi_{t} 1_{[0,+\infty)}(t)(t \geq 0)$,

Then $X, \mu$, and $f$ satisfy all the assumptions of Theorem 2.2, and so we obtain a progressively measurable process $\xi$ with upper-right continuous paths such that

$$
\lambda e^{-\beta T} \psi_{T} 1_{\{T<+\infty\}}=\mathbb{E}\left[\int_{(T,+\infty]} f\left(t, \sup _{v \in[T, t)} \xi_{v}\right) \mu(d t) \mid \mathscr{F}_{T}\right]
$$

for any stopping time $T \in \mathscr{T}$. We shall show below that $\xi<0$ on $[0,+\infty)$ almost surely. Thus, the preceding equation reduces to

$$
\lambda e^{-\beta T} \psi_{T} 1_{\{T<+\infty\}}=\mathbb{E}\left[\int_{(T,+\infty]} \beta e^{-\beta t} \partial_{y} u\left(t,-e^{-\beta t} / \sup _{v \in[T, t)} \xi_{v}\right) \nu(d t) \mid \mathscr{F}_{T}\right] .
$$


Hence, the representation problem 16 is solved by the process $L_{v} \triangleq-1 /\left(\xi_{v} e^{\beta v}\right)>$ $0(v \in[0,+\infty))$.

In order to prove our claim that $\xi<0$ on $[0,+\infty)$ almost surely, consider the stopping time

$$
\tilde{T} \triangleq \inf \left\{t \geq 0 \mid \xi_{t} \geq 0\right\}
$$

On $\{\tilde{T}<+\infty\}$ upper right continuity of $\xi$ implies $\xi_{\tilde{T}} \geq 0$ almost surely. Thus, choosing $T=\tilde{T}$ in the above representation, we obtain by definition of $f$ :

$$
\lambda e^{-\beta \tilde{T}} \psi_{\tilde{T}} 1_{\{\tilde{T}<+\infty\}}=-\mathbb{E}\left[\int_{(\tilde{T},+\infty]} 0 \vee \sup _{v \in[\tilde{T}, t)} \xi_{v} \mu(d t) \mid \mathscr{F}_{\tilde{T}}\right] .
$$

Obviously, the right side in this equality is $\leq 0$ almost surely while its left side is $>0$ except on $\{\tilde{T}=+\infty\}$ where it is 0 . It follows that $\mathbb{P}[\tilde{T}=+\infty]=1$, i.e., $\xi<0$ on $[0,+\infty) \mathbb{P}-$ a.s.

In order to illustrate the role of the representation theorem, let us have a closer look at the case of an American put option as discussed in Theorem 1.8. The decision to exercise an American put option involves a tradeoff between the sure proceeds one can realize immediately and the uncertain future prospects offered by the option. This tradeoff is determined by two factors. Firstly, one has to account for the downward risk in the future evolution of the underlying: If the price process is likely to fall in the near future, one would prefer to wait and exercise the option later. Secondly, one faces a deadline: The option holder can only benefit from the option up to its maturity $\hat{T}$, and so waiting for lower prices bears the risk of not being able to exercise the option at all. The tradeoff between these competing aspects of American puts is reflected in the following characterization of the universal exercise signal $K=\left(K_{t}\right)_{t \in[0, \hat{T}]}$ which is derived from Theorem 2.2. In fact, for American puts in a model with constant interest rates $r>0$, the characterization (22) and the arguments for Corollary 2.4 yield that

$$
\begin{aligned}
K_{S} & =\operatorname{ess}_{T \in \mathscr{T}((S,+\infty])} \frac{\mathbb{E}\left[e^{-r S} P_{S}-e^{-r T} P_{T \wedge \hat{T}} \mid \mathscr{F}_{S}\right]}{\mathbb{E}\left[\int_{(S, T]} r e^{-r t} d t \mid \mathscr{F}_{S}\right]} \\
& =\operatorname{ess}_{T \in \mathscr{T}((S,+\infty])} \frac{\mathbb{E}\left[P_{S}-e^{-r(T-S)} P_{T \wedge \hat{T}} \mid \mathscr{F}_{S}\right]}{\mathbb{E}\left[1-e^{-r(T-S)} \mid \mathscr{F}_{S}\right]}
\end{aligned}
$$

for all stopping times $S \in \mathscr{T}([0, \hat{T}])$. It follows that $K_{S}>k$ iff there is a stopping time $T>S$ such that

$$
\mathbb{E}\left[P_{S}-e^{-r(T-S)} P_{T \wedge \hat{T}} \mid \mathscr{F}_{S}\right]>k \mathbb{E}\left[1-e^{-r(T-S)} \mid \mathscr{F}_{S}\right]
$$


or, equivalently,

$$
k-P_{S}<\mathbb{E}\left[e^{-r(T-S)}\left(k-P_{T \wedge \hat{T}}\right) \mid \mathscr{F}_{S}\right] \leq \mathbb{E}\left[e^{-r(T-S)}\left(k-P_{T \wedge \hat{T}}\right)^{+} \mid \mathscr{F}_{S}\right] .
$$

Hence, $K_{S}>k$ means that exercising the put option with strike $k$ should be postponed since there is an opportunity for stopping later than $S$ which makes us expect a higher discounted payoff. This provides another intuitive explanation why $K_{S}$ should be viewed as a universal exercise signal. However, using formula 25) in order to compute $K_{S}$ amounts to solving a non-standard optimal stopping problem for a quotient of two expectations. Such stopping problems are hard to solve directly. Morimoto (1991) uses a Lagrange multiplier technique in order to reduce this non-standard problem to the solution of a family of standard optimal stopping problems. In the context of American options, this is as complex as the initially posed problem of optimally exercising the American put with arbitrary strike. In contrast, our characterization of $K_{S}$ via the representation problem (2.1) provides a possibility to compute $K_{S}$ without solving any optimal stopping problems, as illustrated by the case studies in Section 3 .

\subsection{Proof of existence and uniqueness}

Let us now discuss the proof of Theorem 2.2, following the arguments of Bank and El Karoui (2002). We start with the uniqueness part and prove the characterization

$$
\xi_{S}=\underset{T \in \mathscr{T}((S,+\infty])}{\operatorname{essinf}} \Xi_{S, T} \quad \text { for any } \quad S \in \mathscr{T}([0,+\infty))
$$

with $\Xi_{S, T}$ as in $(23)$.

In order to show that ' $\leq$ ' holds true, consider a stopping time $T \in \mathscr{T}((S,+\infty])$ and use the representation property of $\xi$ to write

$$
X_{S}=\mathbb{E}\left[\int_{(S, T]} f\left(t, \sup _{v \in[S, t)} \xi_{v}\right) \mu(d t) \mid \mathscr{F}_{S}\right]+\mathbb{E}\left[\int_{(T,+\infty]} f\left(t, \sup _{v \in[S, t)} \xi_{v}\right) \mu(d t) \mid \mathscr{F}_{S}\right] .
$$

As $f(t,$.$) is decreasing, the first f(\ldots)$-term is $\leq f\left(t, \xi_{S}\right)$ and the second one is $\leq$ $f\left(t, \sup _{v \in[T, t)} \xi_{v}\right)$. Hence:

$$
X_{S} \leq \mathbb{E}\left[\int_{(S, T]} f\left(t, \xi_{S}\right) \mu(d t) \mid \mathscr{F}_{S}\right]+\mathbb{E}\left[\int_{(T,+\infty]} f\left(t, \sup _{v \in[T, t)} \xi_{v}\right) \mu(d t) \mid \mathscr{F}_{S}\right] .
$$

Using the representation property of $\xi$ again, we can rewrite the second conditional expectation as

$$
\mathbb{E}\left[\int_{(T,+\infty]} f\left(t, \sup _{v \in[T, t)} \xi_{v}\right) \mu(d t) \mid \mathscr{F}_{S}\right]=\mathbb{E}\left[X_{T} \mid \mathscr{F}_{S}\right]
$$


It follows by definition of $\Xi_{S, T}$ that

$$
\mathbb{E}\left[\int_{(S, T]} f\left(t, \Xi_{S, T}\right) \mu(d t) \mid \mathscr{F}_{S}\right]=\mathbb{E}\left[X_{S}-X_{T} \mid \mathscr{F}_{S}\right] \leq \mathbb{E}\left[\int_{(S, T]} f\left(t, \xi_{S}\right) \mu(d t) \mid \mathscr{F}_{S}\right] .
$$

As both $\Xi_{S, T}$ and $\xi_{S}$ are $\mathscr{F}_{S}$-measurable, this implies that $\xi_{S} \leq \Xi_{S, T}$ almost surely.

In order to show that $\xi_{S}$ is the largest larger lower bound on the family $\Xi_{S, T}, T \in$ $\mathscr{T}((S,+\infty])$, consider the sequence of stopping times

$$
T^{n} \triangleq \inf \left\{t \in(S,+\infty] \mid \sup _{v \in[S, t)} \xi_{v}>\eta_{n}\right\} \quad(n=1,2, \ldots)
$$

where

$$
\eta_{n}=\left(\xi_{S}+1 / n\right) 1_{\left\{\xi_{S}>-\infty\right\}}-n 1_{\left\{\xi_{S}=-\infty\right\}} .
$$

Observe that pathwise upper-right continuity of $\xi$ implies $T^{n} \in \mathscr{T}((S,+\infty])$ and also

$$
\sup _{v \in[S, t)} \xi_{v}=\sup _{\left[T^{n}, t\right)} \xi_{v} \quad \text { for all } \quad t \in\left(T^{n},+\infty\right] \quad \mathbb{P}-\text { a.s. }
$$

since $T^{n}$ is a time of increase for $t \mapsto \sup _{v \in[S, t)} \xi_{v}$. Thus, we obtain

$$
\begin{aligned}
X_{S} & =\mathbb{E}\left[\int_{\left(S, T^{n}\right]} f\left(t, \sup _{v \in[S, t)} \xi_{v}\right) \mu(d t) \mid \mathscr{F}_{S}\right]+\mathbb{E}\left[\int_{\left(T^{n},+\infty\right]} f\left(t, \sup _{v \in\left[T^{n}, t\right)} \xi_{v}\right) \mu(d t) \mid \mathscr{F}_{S}\right] \\
\geq & \mathbb{E}\left[\int_{\left(S, T^{n}\right]} f\left(t, \eta_{n}\right) \mu(d t) \mid \mathscr{F}_{S}\right]+\mathbb{E}\left[X_{T^{n}} \mid \mathscr{F}_{S}\right],
\end{aligned}
$$

where the last estimate follows from our definition of $T^{n}$ and from the representation property of $\xi$ at time $T^{n}$. As $\eta_{n}$ is $\mathscr{F}_{S}$-measurable, the above estimate implies

$$
\eta_{n} \geq \Xi_{S, T^{n}} \geq \operatorname{essinf}_{T \in \mathscr{T}((S,+\infty])} \Xi_{S, T}
$$

Now note that for $n \uparrow+\infty$, we have $\eta_{n} \downarrow \xi_{S}$, and so we obtain the converse inequality ' $\geq$ ' in 26 .

Let us now turn to the existence part of Theorem 2.2, and let us sketch the construction of a solution $\xi$ to the representation problem 2.1 for the technical details we refer to Bank and El Karoui (2002).

The definition of Gittins indices 20) and their representation property (21) suggest to consider the family of optimal stopping problems

$$
Y_{S}^{x}=\underset{T \in \mathscr{T}([S,+\infty])}{\operatorname{essinf}} \mathbb{E}\left[X_{T}+\int_{(S, T]} f(t, x) \mu(d t) \mid \mathscr{F}_{S}\right] \quad(S \in \mathscr{T}, x \in \mathbb{R})
$$


and to define the process $\xi$ as

$$
\xi_{t}(\omega) \triangleq \max \left\{x \in \mathbb{R} \cup\{-\infty\} \mid Y_{t}^{x}(\omega)=X_{t}(\omega)\right\} \quad(t \in[0,+\infty), \omega \in \Omega) .
$$

Since $\mu$ has no atoms, we can use results from El Karoui (1981) to choose a 'nice' version of the random field $Y=\left(Y_{S}^{x}\right)$ such that $\xi$ is an optional process and such that for any $x \in \mathbb{R}, S \in \mathscr{T}$ the stopping time

$$
T_{S}^{x} \triangleq \inf \left\{t \geq S \mid Y_{t}^{x}=X_{t}\right\} \in \mathscr{T}([S,+\infty])
$$

attains the essential infimum in (27):

$$
Y_{S}^{x}=\mathbb{E}\left[X_{T_{S}^{x}}+\int_{\left(S, T_{S}^{x}\right]} f(t, x) \mu(d t) \mid \mathscr{F}_{S}\right] .
$$

For any $S \in \mathscr{T}, Y_{S}^{x}$ is dominated by $X_{S}$ and continuously decreasing in $x$ with $\lim _{x \downarrow-\infty} Y_{S}^{x}=X_{S}$ almost surely. The key observation is that the corresponding negative random measure $Y_{S}(d x)$ can be disintegrated in the form

$$
Y_{S}(d x)=\mathbb{E}\left[\int_{(S,+\infty]}\left\{\int_{-\infty}^{+\infty} 1_{\left(S, T_{S}^{x}\right]}(t) f(t, d x)\right\} \mu(d t) \mid \mathscr{F}_{S}\right],
$$

where $f(t, d x)$ is the negative measure induced by the decreasing function $x \mapsto f(t, x)$. This disintegration formula can be viewed as a generalization of Lemma 2.3 in El Karoui and Karatzas (1994) to the nonlinear case; compare also Lemma 2 in Whittle (1980) for a discrete-time analogue in a Markovian setting.

Using the definition of $\xi_{S}$, this allows us to write for any $y \in \mathbb{R}$ :

$$
\begin{aligned}
X_{S} & =Y_{S}^{\xi_{S}}=Y_{S}^{y}-\int_{\xi_{S} \wedge y}^{y} Y_{S}(d x) \\
& =Y_{S}^{y}-\mathbb{E}\left[\int_{(S,+\infty]}\left\{\int_{-\infty}^{+\infty} 1_{\left(S, T_{S}^{x}\right]}(t) 1_{\left[\xi_{S} \wedge y, y\right)}(x) f(t, d x)\right\} \mu(d t) \mid \mathscr{F}_{S}\right]
\end{aligned}
$$

By definition of $T_{S}^{x}$ and $\xi$, we have

$$
\begin{aligned}
\left\{(\omega, t, x) \mid T_{S}^{x} \geq t\right\} & =\left\{(\omega, t, x) \mid Y_{v}^{x}<X_{v} \text { for all } v \in[S, t)\right\} \\
& =\left\{(\omega, t, x) \mid x>\xi_{v} \text { for all } v \in[S, t)\right\} \\
& =\left\{(\omega, t, x) \mid x \geq \sup _{v \in[S, t)} \xi_{v}\right\}
\end{aligned}
$$


up to a $\mathbb{P} \otimes \mu(d t) \otimes f(t, d x)$-null set. Hence, the above conditional expectation simplifies to

$$
\begin{aligned}
& \mathbb{E}\left[\int_{(S,+\infty]}\left\{\int_{-\infty}^{+\infty} 1_{\left(S, T_{S}^{x}\right]}(t) 1_{\left[\xi_{S} \wedge y, y\right)}(x) f(t, d x)\right\} \mu(d t) \mid \mathscr{F}_{S}\right] \\
& =\mathbb{E}\left[\int_{(S,+\infty]}\left\{\int_{-\infty}^{+\infty} 1_{\left[\sup _{v \in[S, t)} \xi_{v},+\infty\right)}(x) 1_{\left[\xi_{S} \wedge y, y\right)}(x) f(t, d x)\right\} \mu(d t) \mid \mathscr{F}_{S}\right] \\
& =\mathbb{E}\left[\int_{(S,+\infty]}\left\{f(t, y)-f\left(t, \sup _{v \in[S, t)} \xi_{v} \wedge y\right)\right\} \mu(d t) \mid \mathscr{F}_{S}\right] \\
& =\mathbb{E}\left[\int_{\left(S, T_{S}^{x}\right]}\left\{f(t, y)-f\left(t, \sup _{v \in[S, t)} \xi_{v}\right)\right\} \mu(d t) \mid \mathscr{F}_{S}\right]
\end{aligned}
$$

where the last equality holds true since $f(t, y)=f\left(t, \sup _{v \in[S, t)} \xi_{v} \wedge y\right)$ on $\left\{\sup _{v \in[S, t)} \xi_{v}>\right.$ $y\}=\left\{T_{S}^{y}<t\right\}$.

Plugging this equation into the above representation of $X_{S}$ we obtain

$$
\begin{aligned}
X_{S} & =Y_{S}^{y}-\mathbb{E}\left[\int_{\left(S, T_{S}^{y}\right]}\left\{f(t, y)-f\left(t, \sup _{v \in[S, t)} \xi_{v}\right)\right\} \mu(d t) \mid \mathscr{F}_{S}\right] \\
& =\mathbb{E}\left[X_{T_{S}^{y}} \mid \mathscr{F}_{S}\right]+\mathbb{E}\left[\int_{\left(S, T_{S}^{y}\right]} f\left(t, \sup _{v \in[S, t)} \xi_{v}\right) \mu(d t) \mid \mathscr{F}_{S}\right]
\end{aligned}
$$

where the second equality follows from (29) for $x=y$. Letting $y \uparrow+\infty$ in (30), we deduce the desired representation

$$
X_{S}=\mathbb{E}\left[\int_{(S,+\infty]} f\left(t, \sup _{v \in[S, t)} \xi_{v}\right) \mu(d t) \mid \mathscr{F}_{S}\right] .
$$

\section{Explicit solutions}

Let us now provide explicit solutions to the representation problem discussed in the previous section in some specific models with strong homogeneity properties.

\subsection{Lévy models}

In this section, we consider two situations where the source of randomness is modelled as a Lévy process $Y=\left(Y_{t}\right)_{t \in[0,+\infty)}$, defined as a right continuous process whose increments $Y_{t}-Y_{s}, s \leq t$, are independent of $\mathscr{F}_{s}$ and have the same distribution as $Y_{t-s}$; see Bertoin (1996). As classical examples, this includes Brownian motions and Poisson processes with constant drift. But there is a rich variety of other Lévy models appearing in Finance; see, e.g., Eberlein and Keller (1995), Barndorff-Nielsen (1998). 


\subsubsection{The perpetual American put}

We shall start our illustration by considering a perpetual American put on an underlying process $P$ which takes the form

$$
P_{t}=p \exp \left(Y_{t}\right) \quad(t \geq 0)
$$

for some initial price $p>0$ and some Lévy process $Y$. Let us assume that interest rates are given by a constant $r>0$. In this case, Theorem 1.8 suggests to consider the representation problem

$$
e^{-r T} P_{T}=\mathbb{E}\left[\int_{(T,+\infty]} r e^{-r t} \inf _{v \in[T, t)} K_{v} d t \mid \mathscr{F}_{T}\right] \quad(T \in \mathscr{T}) .
$$

This problem can be solved explicitly:

Lemma 3.1 The process $K_{v}=P_{v} / \kappa(v \geq 0)$ with

$$
\kappa \triangleq \mathbb{E}\left[\int_{(0,+\infty]} r e^{-r t} \inf _{v \in[0, t)} \exp \left(Y_{v}\right) d t\right] \in(0,1)
$$

solves the representation problem (32) for the perpetual American put.

Proof : Take a stopping time $T \in \mathscr{T}([0,+\infty])$, and use the Ansatz $K_{v}=P_{v} / \kappa$ with $\kappa>0$ to rewrite the right side of $(32)$ as

$$
\begin{aligned}
\mathbb{E} & {\left[\int_{(T,+\infty]} r e^{-r t} \inf _{v \in[T, t)} K_{v} d t \mid \mathscr{F}_{T}\right] } \\
& =\mathbb{E}\left[\int_{(T,+\infty]} r e^{-r t} \inf _{v \in[T, t)}\left\{p \exp \left(Y_{v}\right) / \kappa\right\} d t \mid \mathscr{F}_{T}\right] \\
& =p e^{-r T} \exp \left(Y_{T}\right) \mathbb{E}\left[\int_{(T,+\infty]} r e^{-r(t-T)} \inf _{v \in[T, t)} \exp \left(Y_{v}-Y_{T}\right) d t \mid \mathscr{F}_{T}\right] / \kappa \\
& =e^{-r T} P_{T} \mathbb{E}\left[\int_{(0,+\infty]} r e^{-r t} \inf _{v \in[0, t)} \exp \left(Y_{v}\right) d t\right] / \kappa
\end{aligned}
$$

where for the last equality we used that $Y$ is a Lévy process. Now, choosing $\kappa$ as in the formulation of the present lemma yields the solution to (32).

It follows from Theorem 1.8 that an investor using (31) as a model for the underlying will exercise a perpetual American put with strike $k>0$ at time

$$
\underline{T}^{k}=\inf \left\{t \geq 0 \mid K_{t} \leq k\right\}=\inf \left\{t \geq 0 \mid P_{t} \leq \kappa k\right\} .
$$


i.e., when the underlying's price has fallen below $100 \times \kappa \%$ of the strike. This result also appears in Mordecki (2002), but the proof is different: It reduces the problem to a classical result on optimal stopping rules for geometric random walks by Darling, Liggett, and Taylor (1972); see also Asmussen, Avram, and Pistorius (2002) and Boyarchenko and Levendorskiu (2002).

\subsubsection{Optimal consumption}

In the context of optimal consumption choice as discussed in Section 1.2 and under appropriate homogeneity assumptions, the arguments for obtaining Lemma 3.1 yield an explicit representation for the discounted price deflator $\left(e^{-\beta t} \psi_{t}\right)_{t \in[0,+\infty)}$. In fact, suppose that the deflator $\psi$ takes the form of an exponential Lévy process,

$$
\psi_{t}=\exp \left(Y_{t}\right) \quad(t \in[0,+\infty)),
$$

and that the agent's utility function $u(t, y)$ is constant over time and of the HARA form

$$
u(t, y)=\frac{y^{\alpha}}{\alpha} \quad(t, y \in[0,+\infty))
$$

for some parameter of risk aversion $\alpha \in(0,1)$. Furthermore, assume a homogeneous time preference structure specified by $\nu(d t) \triangleq \delta e^{-\delta t} d t$ for some constant $\delta>0$. Then the representation problem (16) of Theorem 1.15 reads

$$
\begin{aligned}
\lambda e^{-\beta T} \psi_{T} & =\mathbb{E}\left[\int_{(T,+\infty]} \partial_{y} u\left(\sup _{v \in[T, t)}\left\{L_{v} e^{\beta(v-t)}\right\}\right) \beta \delta e^{-(\beta+\delta) t} d t \mid \mathscr{F}_{T}\right] \\
& =\mathbb{E}\left[\int_{(T,+\infty]} \delta e^{-(\alpha \beta+\delta) t} \inf _{v \in[T, t)}\left\{L_{v}^{\alpha-1} e^{\beta(\alpha-1) v}\right\} d t \mid \mathscr{F}_{T}\right]
\end{aligned}
$$

with $T \in \mathscr{T}$. Since $\psi$ is an exponential Lévy process, this is essentially the same representation problem as discussed in Lemma 3.1. We can therefore identify the solution to (16) as the process $L$ given by

$$
L_{v}=\left(e^{\delta t} \psi_{t}\right)^{-\frac{1}{1-\alpha}} / \kappa \quad(v \in[0,+\infty))
$$

for some constant $\kappa>0$. Hence, the minimal level process is again an exponential Lévy process. It now follows from the description of optimal consumption plans given in Theorem 1.15 and Equation (17) that the qualitative behavior of the consumption process is the same as the behavior of the running supremum of such an exponential Lévy process. 
In the economic interpretation, this implies that a variety of different consumption patterns can be optimal, depending on the underlying stochastic model. If, for instance, $\psi$ is an exponential Poisson process with drift, consumption will occur in gulps whenever there is a favorable downward price shock. Consumption at rates occurs in models where the deflator is driven by a Lévy process without downward jumps and with vanishing diffusion part. If, on the other hand, the price deflator $\psi$ is specified as a geometric Brownian motion, consumption occurs in a singular way, similar to the behavior of Brownian local time. For a more detailed study of optimal consumption behavior, including a discussion of the corresponding investment strategies, we refer to Bank (2000) and Bank and Riedel (2001).

\subsection{Diffusion models}

Let $X=\left(X_{t}\right)_{t \in[0,+\infty)}$ be specified as a time-homogeneous one-dimensional diffusion with state space $(0,+\infty)$, and let $\mathbb{P}_{x}$ denote its distribution when started in $x \in(0,+\infty)$. An application of the strong Markov property shows that the Laplace transforms of the level passage times

$$
T_{y}=\inf \left\{t \geq 0 \mid X_{t}=y\right\}
$$

satisfy

$$
\mathbb{E}_{x} e^{-r T_{z}}=\mathbb{E}_{x} e^{-r T_{y}} \mathbb{E}_{y} e^{-r T_{z}} \quad \text { for any } \quad x>y>z \geq 0, r>0 .
$$

Hence, these Laplace transforms are of the form

$$
\mathbb{E}_{x} e^{-r T_{y}}=\frac{\varphi_{r}(x)}{\varphi_{r}(y)} \quad(x>y>0)
$$

for some continuous and strictly decreasing function $\varphi_{r}:(0,+\infty) \rightarrow(0,+\infty)$ with $\varphi_{r}(y) \uparrow+\infty$ as $y \downarrow 0$; we refer to Itô and McKean (1965) for a detailed discussion.

Lemma 3.2 If the function $\varphi_{r}$ of (33) is strictly convex and continuously differentiable, the solution $\xi=\left(\xi_{v}\right)_{v \in[0,+\infty)}$ of the representation problem

$$
e^{-r T} X_{T} 1_{\{T<+\infty\}}=\mathbb{E}\left[\int_{(T,+\infty]} r e^{-r t} \inf _{v \in[T, t)} \xi_{v} d t \mid \mathscr{F}_{T}\right] \quad(T \in \mathscr{T})
$$

takes the form $\xi_{v}=\kappa\left(X_{v}\right)$ where the function $\kappa$ is given by

$$
\kappa(x) \triangleq x-\frac{\varphi_{r}(x)}{\varphi_{r}^{\prime}(x)} \quad(x \in(0,+\infty)) .
$$


Proof : We choose the Ansatz $\xi_{v}=\kappa\left(X_{v}\right)$, where $\kappa$ is a continuous function on $(0,+\infty)$. Using the strong Markov property, we see that the representation problem 34 amounts to specifying $\kappa$ such that

$$
x=\mathbb{E}_{x} \int_{0}^{+\infty} r e^{-r t} \inf _{v \in[0, t)} \kappa\left(X_{v}\right) d t \quad \text { for all } \quad x \in[0,+\infty) .
$$

Equivalently, we can write

$$
x=\mathbb{E}_{x} \inf _{v \in\left[0, \tau_{r}\right)} \kappa\left(X_{v}\right) \text { for all } x \in[0,+\infty)
$$

where $\tau_{r}$ denotes an independent, exponentially distributed random time with parameter $r$. If we assume that $\kappa$ is strictly increasing with $\kappa(0+)=0$ and $\kappa(+\infty)=+\infty$, then the right side in $(36)$ can be rewritten as

$$
\mathbb{E}_{x}\left[\inf _{v \in\left[0, \tau_{r}\right)} \kappa\left(X_{v}\right)\right]=\mathbb{E}_{x}\left[\kappa\left(\inf _{v \in\left[0, \tau_{r}\right)} X_{v}\right)\right]=\int_{0}^{+\infty} \mathbb{P}_{x}\left[\kappa\left(\inf _{v \in\left[0, \tau_{r}\right)} X_{v}\right)>y\right] d y
$$

We have

$$
\begin{aligned}
\mathbb{P}_{x}\left[\kappa\left(\inf _{v \in\left[0, \tau_{r}\right)} X_{v}\right)>y\right] & =\mathbb{P}_{x}\left[\inf _{v \in\left[0, \tau_{r}\right)} X_{v}>\kappa^{-1}(y)\right] \\
& = \begin{cases}0 & \text { if } x \leq \kappa^{-1}(y), \text { i.e., } y \geq \kappa(x), \\
\mathbb{P}_{x}\left[T_{\kappa^{-1}(y)}>\tau_{r}\right] & \text { otherwise. }\end{cases}
\end{aligned}
$$

By Fubini's theorem,

$$
\begin{aligned}
\mathbb{P}_{x}\left[T_{\kappa^{-1}(y)}>\tau_{r}\right] & =\int_{0}^{+\infty} r e^{-r t}\left\{\int_{t}^{+\infty} \mathbb{P}_{x}\left[T_{\kappa^{-1}(y)} \in d s\right]\right\} d t \\
& =\int_{0}^{+\infty}\left\{\int_{0}^{s} r e^{-r t} d t\right\} \mathbb{P}_{x}\left[T_{\kappa^{-1}(y)} \in d s\right] \\
& =1-\mathbb{E}_{x} e^{-r T_{\kappa^{-1}(y)}}=1-\frac{\varphi_{r}(x)}{\varphi_{r}\left(\kappa^{-1}(y)\right)} .
\end{aligned}
$$

Plugging this into (37) yields

$$
\mathbb{E}_{x}\left[\inf _{v \in\left[0, \tau_{r}\right)} \kappa\left(X_{v}\right)\right]=\int_{0}^{\kappa(x)}\left\{1-\frac{\varphi_{r}(x)}{\varphi_{r}\left(\kappa^{-1}(y)\right)}\right\} d y=\kappa(x)-\varphi_{r}(x) \int_{0}^{x} \frac{d \kappa(z)}{\varphi_{r}(z)},
$$

where we use the substitution $y=\kappa(z)$ in the last step. Combining this with (36) shows that $\kappa$ satisfies

$$
\kappa(x)=x+\varphi_{r}(x) \int_{0}^{x} \frac{d \kappa(z)}{\varphi_{r}(z)} \quad(x \in(0,+\infty)) .
$$


Writing this identity in differential form yields

$$
d \kappa(x)=d x+d \varphi_{r}(x) \int_{0}^{x} \frac{d \kappa(z)}{\varphi_{r}(z)}+d \kappa(x)
$$

or, equivalently,

$$
d \kappa(x)=-\varphi_{r}(x) d \frac{1}{\varphi_{r}^{\prime}(x)} .
$$

Thus,

$$
\kappa(x)=-\int_{0}^{x} \varphi_{r}(y) d \frac{1}{\varphi_{r}^{\prime}(y)}=x-\frac{\varphi_{r}(x)}{\varphi_{r}^{\prime}(x)} \quad(x \in(0,+\infty)),
$$

where the last equality follows by partial integration; note that $\lim _{y \downarrow 0} \varphi_{r}(y) / \varphi_{r}^{\prime}(y)=0$ by convexity of $\varphi_{r}$. Since $\varphi_{r}$ is strictly convex with continuous derivative by assumption, this function $\kappa$ is in fact strictly increasing, continuous and surjective. Hence, the preceding calculations are justified, and so we have shown that the function $\kappa$ defined in (35) satisfies (36) as desired.

The explicit solution derived in Lemma 3.2 can readily be applied to the different optimization problems discussed in Section 1. In fact, this result is closely related to the explicit computation of Gittins indices for one-dimensional diffusions as carried out in Karatzas (1984) and El Karoui and Karatzas (1994). Note, however, that their calculation is based on the characterization of Gittins indices as essential infima over certain stopping times, while our argument identifies the function $\kappa$ directly as the solution of the representation problem (36).

\section{Algorithmic aspects}

Closed-form solutions as derived in the previous sections are typically available only under strong homogeneity assumptions. In practice, however, one usually has to face inhomogeneities. One important example in Finance is the American put with finite time horizon which does not allow for closed-form solutions even in the simplest case of the Black-Scholes model. In order to deal with such inhomogeneous problems, it becomes necessary to use computational methods. For this reason, let us focus on some algorithmic aspects of our general representation problem (2.1) in a discrete-time setting, following Bank (2003a).

Specifically, we assume that $\mu$ is given as a sum of Dirac measures

$$
\mu(d t)=\sum_{i=1}^{n+1} \delta_{t_{i}}(d t)
$$


so that

$$
\mathbb{T} \triangleq \operatorname{supp} \mu \cup\{0\}=\left\{0 \triangleq t_{0}<t_{1}<\ldots<t_{n+1} \triangleq+\infty\right\}
$$

is finite. Suppose that, for any $t=t_{1}, \ldots, t_{n+1}$, the function $f=f(\omega, t, x)$ is continuously and strictly decreasing from $+\infty$ to $-\infty$ in $x \in \mathbb{R}$ with

$$
f(t, x) \in L^{1}\left(\Omega, \mathscr{F}_{t}, \mathbb{P}\right) .
$$

In this situation the construction of a solution to the discrete-time version

$$
X_{T}=\mathbb{E}\left[\sum_{s \in \mathbb{T}, s>T} f\left(s, \max _{v \in \mathbb{T} \cap[t, s)} \xi_{v}\right) \mid \mathscr{F}_{T}\right] \quad(T \in \mathscr{T}(\mathbb{T})) .
$$

of our representation problem becomes straightforward.

In fact, there are several rather obvious ways to compute the solution $\xi=$ $\left(\xi_{t}\right)_{t \in \mathbb{T} \cap[0,+\infty)}$. One approach is by backwards induction: First solve for $\xi_{t_{n}}$ in

$$
X_{t_{n}}=\mathbb{E}\left[f\left(t_{n+1}, \xi_{t_{n}}\right) \mid \mathscr{F}_{t_{n}}\right]
$$

and then, having constructed $\xi_{t_{n}}, \xi_{t_{n-1}}, \ldots, \xi_{t_{i+1}}$, compute $\xi_{t_{i}}$ as the unique solution $\Xi \in L^{0}\left(\mathscr{F}_{t_{i}}\right)$ to the equation

$$
X_{t_{i}}=\mathbb{E}\left[f\left(t_{i+1}, \Xi\right)+\sum_{s \in \mathbb{T}, s>t_{i+1}} f\left(s, \Xi \vee \max _{v \in \mathbb{T} \cap\left[t_{i+1}, s\right)} \xi_{v}\right) \mid \mathscr{F}_{t_{i}}\right] .
$$

However, this approach may be tedious from a computational point of view. In fact, solving for $\Xi$ in the above equation amounts to solving a highly nonlinear equation given in terms of a conditional expectation of a path-dependent random variable.

As an alternative, one might use the characterization of $\xi$ in Theorem 2.2 and compute

$$
\underset{T \in \mathscr{T}\left(\mathbb{T} \cap\left(t_{i},+\infty\right]\right)}{\operatorname{essinf}} \Xi_{t, T}
$$

for each $t \in \mathbb{T} \cap[0,+\infty)$, where $\Xi_{t, T}$ denotes the unique solution $\Xi \in L^{0}\left(\mathscr{F}_{t}\right)$ to

$$
\mathbb{E}\left[X_{t}-X_{T} \mid \mathscr{F}_{t}\right]=\mathbb{E}\left[\sum_{s \in \mathbb{T}, s>t} f(s, \Xi) \mid \mathscr{F}_{t}\right] .
$$

Solving for $\Xi$ in this equation is comparably easy. For instance, in the separable case $f(s, x)=g(s) h(x)$ one finds

$$
\Xi_{t, T}=h^{-1}\left(\frac{\mathbb{E}\left[X_{t}-X_{T} \mid \mathscr{F}_{t}\right]}{\mathbb{E}\left[\sum_{s \in \mathbb{T}, s>t} g(s) \mid \mathscr{F}_{t}\right]}\right) .
$$


A crucial drawback of this approach, however, is that the class of stopping times $\mathscr{T}(\mathbb{T} \cap$ $(t,+\infty])$ is typically huge. Hence, it would be convenient to reduce the number of stopping times $T$ to be considered. This is achieved by the following

\section{Algorithm 4.1}

$$
\begin{aligned}
& \text { AdaptedProcess } \xi ; \xi_{+\infty}=+\infty ; \\
& \text { for (int } i=n, i \geq 0, i=i-1)\{ \\
& \quad \text { StoppingTime } T=t_{i+1} ; \\
& \text { while }\left(\mathbb{P}\left[\Xi_{t_{i}, T}>\xi_{T}\right]>0\right)\{ \\
& \quad T=\min \left\{t \in \mathbb{T} \cap(T,+\infty] \mid \xi_{t} \geq \xi_{T}\right\} \\
& \quad \text { on }\left\{\xi_{T}=\mathscr{F}_{t_{i}}-\operatorname{essinf} \xi_{T}<\Xi_{t_{i}, T}\right\} ; \\
& \quad\} ; \quad \xi_{t_{i}}=\Xi_{t_{i}, T} ;
\end{aligned}
$$

Here $\mathscr{F}_{t}-$ ess $\inf \xi_{T}$ denotes the largest $\mathscr{F}_{t}-$ measurable random variable which is almost surely dominated by $\xi_{T}$ :

$$
\mathscr{F}_{t}-\operatorname{ess} \inf \xi_{T}=\operatorname{ess} \sup \left\{\Xi \in L^{0}\left(\mathscr{F}_{t}\right) \mid \Xi \leq \xi_{T} \mathbb{P}-\text { a.s. }\right\}
$$

Like the first approach, the algorithm proceeds backwards in time. Similar to the second approach, it constructs the solution $\xi_{t}, t=t_{n}, t_{n-1}, \ldots, t_{0}$, in the form $\xi_{t}=\Xi_{t, T}$. However, instead of considering all stopping times $T \in \mathscr{T}(t,+\infty]$ in order to determine a stopping time with $\xi_{t}=\Xi_{t, T}$, the algorithm constructs an increasing sequence of candidates, starting with the first time in $\mathbb{T}$ after $t$. Step by step, this candidate is carefully updated until the terminal condition $\mathbb{P}\left[\Xi_{t, T}>\xi_{T}\right]=0$ is met.

It follows from the monotonicity of the update rule for $T$ that the algorithm will terminate under

Assumption 4.2 The set of scenarios $\Omega$ is finite.

The main idea of the algorithm is to construct for each $i=n, \ldots, 0$ the stopping time

$$
S_{i}^{*} \triangleq \min \left\{s \in \mathbb{T} \cap\left(t_{i},+\infty\right] \mid \xi_{s} \geq \xi_{t_{i}}\right\}
$$

Since $\mathbb{T}$ is discrete, this stopping time is contained in $\mathscr{T}\left(\mathbb{T} \cap\left(t_{i},+\infty\right]\right)$ and it attains the ess inf in the characterization of $\xi_{t_{i}}$ provided by Theorem 2.2.

Lemma 4.3 For any $t_{i} \in \mathbb{T} \cap[0,+\infty)$, we have

$$
\Xi_{t, S_{i}^{*}}=\xi_{t}=\underset{S \in \mathscr{T}\left(\mathbb{T} \cap\left(t_{i},+\infty\right]\right)}{\operatorname{essinf}} \Xi_{t, S} .
$$


Proof : The first equality is established with the same argument as in the ' $\geq$ '-part of the proof of Theorem 2.2, choosing $T^{n} \equiv S_{i}^{*} \in \mathscr{T}(\mathbb{T} \cap(t,+\infty])$. The second one follows as in Theorem 2.2.

It may seem that the preceding lemma is not of great help for computing $\xi_{t_{i}}$ since $\xi_{t_{i}}$ appears in the definition of $S_{i}^{*}$. However, we are going to show that the stopping time attained upon termination of the while-loop at stage $i$ coincides with $S_{i}^{*}$ even though its construction does not rely on $\xi_{t_{i}}$. This will be the main step in our proof of

Theorem 4.4 Algorithm 4.1 is correct: The resulting process $\xi$ solves the representation problem 38 .

From now on we fix the index $i$ and write $S^{*}=S_{i}^{*}$. Our aim is to prove the identity

$$
S^{*}=T^{*} \quad \mathbb{P}-\text { a.s. }
$$

where $T^{*}$ denotes the value of the algorithm's stopping time $T$ upon termination of the while-loop at stage $i$. As a first step, let us characterize $S^{*}$ in a different way:

Lemma 4.5 The stopping time $S^{*}$ of 39 is minimal among all stopping times $S \in$ $\mathscr{T}\left(t_{i},+\infty\right]$ satisfying $\xi_{S} \geq \Xi_{t_{i}, S}$ almost surely.

Proof : The inequality $\xi_{S^{*}} \geq \Xi_{t_{i}, S^{*}}$ follows immediately from (39) and (40). On the other hand, (40) entails that, for any $S \in \mathscr{T}\left(t_{i},+\infty\right]$ with $\xi_{S} \geq \Xi_{t_{i}, S}$ almost surely, we have $\xi_{S} \geq \xi_{t_{i}}$ almost surely. But this implies $S \geq S^{*} \mathbb{P}$-a.s. by definition of $S^{*}$.

Let us denote the successive instances of the stopping time $T$ during the procession of the while-loop at stage $i$ by $T^{0} \triangleq t_{i+1} \leq T^{1} \leq \ldots \leq T^{*}$ with the convention that $T^{k}=T^{*}$ if the while-loop is processed less than $k$ times. It then follows from the update rule of our algorithm that

$$
\left\{T^{k}<T^{k+1}\right\}=\left\{\xi_{T^{k}}=\mathscr{F}_{t_{i}}-\operatorname{ess} \inf \xi_{T^{k}}<\Xi_{t_{i}, T^{k}}\right\} \quad \mathbb{P}-\text { a.s. }
$$

Since $\Omega$ is finite, the while-loop will be terminated at some point. We thus have $T^{k}=T^{k+1}=T^{*} \mathbb{P}$-a.s. for all sufficiently large $k$. By 42 this means that $T^{*}$ satisfies $\Xi_{t_{i}, T^{*}} \leq \xi_{T^{*}}$ almost surely. In particular, we can infer from Lemma 4.5 that

$$
S^{*} \leq T^{*} \quad \mathbb{P}-\text { a.s. }
$$

Thus, in order to establish our central claim (41), it remains to prove the converse inequality. This is achieved by

Lemma $4.6 T^{k} \leq S^{*}$ almost surely for each $k=0,1, \ldots$ 
Proof : Since $T^{0}=t_{i+1}$ and $S^{*} \geq t_{i+1}$ by definition, our assertion holds true for $k=0$ and so we can proceed by induction. Thus, assume that we already have established $T^{k} \leq S^{*}$ and let us deduce that also $T^{k+1} \leq S^{*}$ almost surely.

To this end, note that on $\left\{T^{k}<S^{*}\right\}$ we have $\xi_{T^{k}}<\xi_{t_{i}} \leq \xi_{S^{*}}$ by definition of $S^{*}$. Since, by definition, $T^{k+1}$ coincides either with $T^{k}$ or with the first time in $\mathbb{T}$ after $T^{k}$ where $\xi$ reaches or exceeds the level $\xi_{T^{k}}$, this implies

$$
T^{k+1} \leq S^{*} \quad \text { almost surely on } \quad\left\{T^{k}<S^{*}\right\} .
$$

Hence, our claim $T^{k+1} \leq S^{*} \mathbb{P}$-a.s. will be proved once we know that

$$
\left\{T^{k}<T^{k+1}\right\} \subset\left\{T^{k}<S^{*}\right\} \quad \text { up to a } \mathbb{P}-\text { null set. }
$$

This inclusion will be established using the following two intermediate results:

(i) Up to a $\mathbb{P}$-null set we have $\left\{\mathbb{P}\left[T^{k}<S^{*} \mid \mathscr{F}_{t_{i}}\right]>0\right\}=\left\{\mathscr{F}_{t_{i}}-\operatorname{ess} \inf \xi_{T^{k}}<\xi_{t_{i}}\right\}$.

Indeed, it follows from $t_{i+1} \leq T^{k} \leq S^{*}$ and the definition of $S^{*}$ that $\left\{T^{k}<S^{*}\right\}=$ $\left\{\xi_{T^{k}}<\xi_{t_{i}}\right\}$ up to a $\mathbb{P}$-null set. Hence,

$$
\mathbb{P}\left[T^{k}<S^{*} \mid \mathscr{F}_{t_{i}}\right]=\mathbb{P}\left[\xi_{T^{k}}<\xi_{t_{i}} \mid \mathscr{F}_{t_{i}}\right] \quad \mathbb{P} \text {-a.s. }
$$

Up to a $\mathbb{P}$-null set, the latter conditional probability is strictly positive if and only if $\mathscr{F}_{t_{i}}-\operatorname{ess} \inf \xi_{T^{k}}<\xi_{t_{i}}$. This proves claim (i).

(ii) Up to a $\mathbb{P}$-null set we have $\left\{\mathbb{P}\left[T^{k}<S^{*} \mid \mathscr{F}_{t_{i}}\right]>0\right\} \supset\left\{\mathscr{F}_{t_{i}}-\operatorname{essinf} \xi_{T^{k}}<\Xi_{t_{i}, T^{k}}\right\}$. Sine $T^{k} \leq S^{*} \mathbb{P}$-a.s. we have that

$$
\begin{aligned}
\left\{\mathbb{P}\left[T^{k}<S^{*} \mid \mathscr{F}_{t_{i}}\right]=0\right\} & =\left\{\mathbb{P}\left[T^{k}=S^{*} \mid \mathscr{F}_{t_{i}}\right]=1\right\} \\
& \subset\left\{\mathbb{P}\left[\xi_{T^{k}}=\xi_{S^{*}} \mid \mathscr{F}_{t_{i}}\right]=1\right\} \\
& \subset\left\{\mathbb{P}\left[\xi_{T^{k}} \geq \Xi_{t_{i}, S^{*}} \mid \mathscr{F}_{t_{i}}\right]=1\right\}
\end{aligned}
$$

up to a $\mathbb{P}$-null set, where the last inclusion holds true since

$$
\xi_{S^{*}} \geq \xi_{t_{i}}=\Xi_{t_{i}, S^{*}} \quad \mathbb{P} \text {-a.s. }
$$

by definition of $S^{*}$ and 407 . Hence, up to a $\mathbb{P}$-null set we can write

$$
\begin{aligned}
\left\{\mathbb{P}\left[T^{k}<S^{*} \mid \mathscr{F}_{t_{i}}\right]=0\right\} & =\left\{\mathbb{P}\left[\xi_{T^{k}} \geq \Xi_{t_{i}, S^{*}} \mid \mathscr{F}_{t_{i}}\right]=1\right\} \cap\left\{\mathbb{P}\left[T^{k}=S^{*} \mid \mathscr{F}_{t_{i}}\right]=1\right\} \\
& \subset\left\{\mathbb{P}\left[\xi_{T^{k}} \geq \Xi_{t_{i}, T^{k}} \mid \mathscr{F}_{t_{i}}\right]=1\right\}
\end{aligned}
$$


where the last inclusion is true as

$$
\left\{\mathbb{P}\left[T^{k}=S^{*} \mid \mathscr{F}_{t_{i}}\right]=1\right\} \subset\left\{\Xi_{t_{i}, S^{*}}=\Xi_{t_{i}, T^{k}}\right\} \in \mathscr{F}_{t_{i}}
$$

by definition of $\Xi_{t_{i},}$.

Passing to complements, the above inclusions imply

$$
\begin{aligned}
\left\{\mathbb{P}\left[T^{k}<S^{*} \mid \mathscr{F}_{t_{i}}\right]>0\right\} & \supset\left\{\mathbb{P}\left[\xi_{T^{k}} \geq \Xi_{t_{i}, T^{k}} \mid \mathscr{F}_{t_{i}}\right]<1\right\} \\
& =\left\{\mathbb{P}\left[\xi_{T^{k}}<\Xi_{t_{i}, T^{k}} \mid \mathscr{F}_{t_{i}}\right]>0\right\} \\
& =\left\{\mathscr{F}_{t_{i}}-\operatorname{essinf} \xi_{T^{k}}<\Xi_{t_{i}, T^{k}}\right\}
\end{aligned}
$$

where the last equality follows from the $\mathscr{F}_{t_{i}}$-measurability of $\Xi_{t_{i}, T^{k}}$ and the definition of $\mathscr{F}_{t_{i}}-\operatorname{ess} \inf \xi_{T^{k}}$.

In order to complete the proof of (43) we use (42), (ii), and (i) to obtain that up to a $\mathbb{P}$-null set we have

$$
\begin{aligned}
\left\{T^{k}<T^{k+1}\right\} & =\left\{\xi_{T^{k}}=\mathscr{F}_{t_{i}}-\operatorname{ess} \inf \xi_{T^{k}}\right\} \cap\left\{\mathscr{F}_{t_{i}}-\operatorname{ess} \inf \xi_{T^{k}}<\Xi_{t_{i}, T^{k}}\right\} \\
& \subset\left\{\xi_{T^{k}}=\mathscr{F}_{t_{i}}-\operatorname{essinf} \xi_{T^{k}}\right\} \cap\left\{\mathbb{P}\left[T^{k}<S^{*} \mid \mathscr{F}_{t_{i}}\right]>0\right\} \\
& =\left\{\xi_{T^{k}}=\mathscr{F}_{t_{i}}-\operatorname{essinf} \xi_{T^{k}}<\xi_{t_{i}}\right\} \\
& \subset\left\{T^{k}<S^{*}\right\}
\end{aligned}
$$

using $T^{k} \leq S^{*} \mathbb{P}$-a.s. and the definition of $S^{*}$ for the last inclusion.

\section{References}

Asmussen, S., F. Avram, and M. Pistorius (2002): "Russian and American put options under exponential phase-type Lvy models," Discussion paper, MaPhySto.

BAnk, P. (2000): "Singular Control of Optional Random Measures - Stochastic Optimization and Representation Problems Arising in the Microeconomic Theory of Intertemporal Consumption Choice," Ph.D. thesis, Humboldt University of Berlin.

(2003a): "An Algorithm for Computing Gittins-Indices and Exercise Signals for American Options," in preparation, Humboldt University of Berlin.

(2003b): "Gittins Indices for American Options," in preparation, Humboldt University of Berlin. 
Bank, P., And N. El Karoui (2002): "A Stochastic Representation Theorem with Applications to Optimization and Obstacle Problems," to appear in The Annals of Probability.

Bank, P., And F. Riedel (2001): "Optimal Consumption Choice with Intertemporal Substitution," Annals of Applied Probability, 3, 750-788.

Barndorff-Nielsen, O. E. (1998): "Processes of normal inverse Gaussian type," Finance Stoch., 2(1), 41-68.

Benth, F. E., K. H. Karlsen, and K. Reikvam (2001): "Optimal portfolio selection with consumption and nonlinear integro-differential equations with gradient constraint: a viscosity solution approach," Finance Stoch., 5(3), 275-303.

Bertoin, J. (1996): Lévy Processes. Cambridge University Press.

Boyarchenko, S. I., And S. Z. LevendorskiI (2002): "Perpetual American options under Lévy processes," SIAM J. Control Optim., 40(6), 1663-1696 (electronic).

Darling, D. A., T. Liggett, and H. M. TAYlor (1972): "Optimal stopping for partial sums," Ann. Math. Statist., 43, 1363-1368.

Delbaen, F., and W. Schachermayer (1994): "A General Version of the Fundamental Theorem of Asset Pricing," Mathematische Annalen, 300, 463-520.

Dellacherie, C., And P. Meyer (1975): Probabilités et potentiel, Chapitres I-IV. Hermann.

(1980): Probabilités et potentiel, Chapitres V-VIII. Hermann.

Eberlein, E., ANd U. Keller (1995): "Hyperbolic Distributions in Finance," Bernoulli, 1, 281-299.

El Karoui, N. (1981): "Les aspects probabilistes du contrôle stochastique," in Ninth Saint Flour Probability Summer School-1979 (Saint Flour, 1979), no. 876 in Lecture Notes in Mathematics, pp. 73-238. Springer, Berlin.

El Karoui, N., and I. Karatzas (1994): "Dynamic Allocation Problems in Continuous Time," Annals of Applied Probability, 4, 255-286.

- (1995): "The optimal stopping problem for a general American put-option," in Mathematical finance, ed. by M. Davis, pp. 63-74. Springer, Berlin, New York. 
(1997): "Synchronization and optimality for multi-armed bandit problems in continuous time," Mat. Apl. Comput., 16(2), 117-151.

El Karoui, N., and M. C. Quenez (1995): "Dynamic Programming and Pricing of Contingent Claims in an Incomplete Market," SIAM Journal on Control and Optimization, 33, 29-66.

Föllmer, H., AND Y. Kabanov (1998): "Optional Decomposition and Lagrange Multipliers," Finance and Stochastics, 2, 69-81.

Föllmer, H., ANd A. Schied (2002): Stochastic Finance: An Introduction in Discrete Time, no. 27 in de Gruyter Studies in Mathematics. de Gruyter.

Gittins, J. C. (1979): "Bandit processes and dynamic allocation indices (with discussion)," J. Roy. Statist. Soc. Ser. B, 41, 148-164.

Hindy, A., And C.-F. Huang (1993): "Optimal Consumption and Portfolio Rules with Durability and Local Substitution," Econometrica, 61, 85-121.

Hindy, A., C.-F. Huang, And D. Kreps (1992): "On intertemporal preferences in continuous time: the case of certainty," J. Math. Econom., 21(5), 401-440.

Itô, K., And H. P. MCKeAn, JR. (1965): Diffusion processes and their sample paths, Die Grundlehren der Mathematischen Wissenschaften, Band 125. Springer, Berlin.

JacoD, J. (1979): Calcul Stochastique et Problèmes de Martingales, no. 714 in Lecture Notes in Mathematics. Springer.

KARAtZAS, I. (1984): "Gittins indices in the dynamic allocation problem for diffusion processes," Ann. Probab., 12(1), 173-192.

Karatzas, I. (1988): "On the Pricing of American Options," App. Math. Optimization, $17,37-60$.

Karatzas, I., and S. E. Shreve (1998): Methods of Mathematical Finance. Springer, New York.

Kramkov, D. (1996): "Optional Decomposition of Supermartingales and Hedging Contingent Claims in Incomplete Security Markets," Probability Theory and Related Fields, 105, 459-479. 
Mandelbaum, A. (1987): "Continuous multi-armed bandits and multiparameter processes," Ann. Probab., 15(4), 1527-1556.

Mordecki, E. (2002): "Optimal Stopping and Perpetual Options for Lévy Processes," Finance and Stochastastics, 6, 473-493.

Morimoto, H. (1991): "On Average Cost Stopping Time Problems," Probability Theory and Related Fields, 90, 469-490.

Snell, J. L. (1952): "Applications of martingale system theorems," Trans. Amer. Math. Soc., 73, 293-312.

Whittle, P. (1980): "Multi-armed bandits and the Gittins index," J. Roy. Statist. Soc. Ser. B, 42(2), 143-149.

Peter Bank

Institut für Mathematik

Humboldt-Universität zu Berlin

Unter den Linden 6

D-10099 Berlin, Germany

pbank@mathematik.hu-berlin.de
HANS FÖLLMER

Institut für Mathematik

Humboldt-Universität zu Berlin

Unter den Linden 6

D-10099 Berlin, Germany

foellmer@mathematik.hu-berlin.de 